Discussion Paper 140

Institute for Empirical Macroeconomics

Federal Reserve Bank of Minneapolis

90 Hennepin Avenue

Minneapolis, Minnesota 55480-0291

Revised May 2004

\title{
Dynamic Optimal Taxation with Private Information
}

\author{
Stefania Albanesi* \\ Duke University \\ Christopher Sleet* \\ University of Iowa
}

\begin{abstract}
We study dynamic optimal taxation in a class of economies with private information. Constrained optimal allocations in these environments are complicated and history-dependent. Yet, we show that they can be implemented as competitive equilibria in market economies supplemented with simple tax systems. The market structure in these economies is similar to that in Bewley (1986): agents supply labor and trade risk-free claims to future consumption, subject to a budget constraint and a debt limit. Optimal taxes are conditioned only on two observable characteristics- an agent's accumulated stock of claims, or wealth, and her current labour income- and they are not additively separable in these variables. The marginal wealth tax is decreasing in labour income and its expected value is generally positive. The marginal labour income tax is decreasing in wealth.
\end{abstract}

\footnotetext{
*Albanesi: albanesi@duke.edu; Sleet: christopher-sleet@uiowa.edu. We thank seminar participants at the University of Chicago, the Federal Reserve Bank of Minneapolis, MIT, Boston University, NYU, Northwestern, Princeton, UBC, UCL, the SED 2003 Annual Meeting, CEPR fiscal policy conference, the 2003 Midwest macro meetings, and SITE for their comments. We are grateful to Marco Bassetto, Paul Beaudry, V.V. Chari, Mike Golosov, John Hassler, Ken Judd, Dirk Krueger, Nicola Pavoni, Fabrizio Perri, Chris Phelan, Gianluca Violante, Ivan Werning and Sevin Yeltekin for helpful discussions. The views expressed herein are those of the authors and not necessarily those of the Federal Reserve Bank of Minneapolis or the Federal Reserve System.
} 


\section{Introduction}

This paper studies optimal taxation in a class of dynamic economies with private information. Specifically, we consider an environment in which agents' preferences are defined over consumption and labour, and each agent receives a privately observed sequence of i.i.d. idiosyncratic shocks. Incentive-compatibility constraints stemming from private information imply that socially optimal, or "constrained efficient", allocations in this environment are complicated and history-dependent. Yet, we show that they can be implemented as competitive equilibria in market economies supplemented with simple tax systems. The market structure in these economies is identical to that in Bewley (1986), Huggett (1993) or Aiyagari (1994): agents can trade current consumption for claims to future consumption, subject to a budget constraint and a borrowing limit. These claims have a non-contingent pre-tax return. Crucially, taxes are conditioned upon only two observable characteristics of an agent: current wealth, given by the agent's accumulated stock of claims, and current labour income. They do not depend on any other aspect of an agent's past history. The model has implications for the optimal taxation of both wealth and labour earnings. It implies optimal taxes that are non-linear and non-separable in these variables. In particular, the marginal tax on wealth is negatively correlated with an agent's labour income, while the marginal labour income tax is decreasing in wealth.

Most models of dynamic optimal taxation follow the Ramsey approach, in which the set of fiscal instruments available to the government is exogenously specified ${ }^{1}$. Linear labour and capital income taxes are typically included in this set, while lump-sum taxes are ruled out. The exclusion of the latter is justified by appealing to incentive or administrative constraints, but these are not explicitly modelled. These exogenous restrictions on fiscal instruments become themselves a source of frictions. The government's optimal taxation problem reduces to one of selecting from amongst a limited number of policy instruments so as to ameliorate these frictions.

The approach we adopt in this paper builds on the optimal non-linear income taxation literature initiated by Mirrlees (1971). This literature emphasises that incentive-compatibility constraints due to private information give rise to endogenous restrictions on optimal tax policies. It then characterises those tax functions that induce agents to select the constrained efficient allocation. The resulting optimal non-linear income taxes reproduce the patterns of wedges - gaps between individual marginal rates of substitution and transformation - induced by the incentive-compatibility constraints at the optimal allocation. This

\footnotetext{
${ }^{1}$ Chari and Kehoe (1999) provide an excellent overview of this literature.
} 
research has concentrated on static models, leaving the properties of optimal taxes in dynamic economies with private information largely unexplored ${ }^{2}$.

We also build on the dynamic contracting literature. ${ }^{3}$ This literature studies the properties of constrainedefficient allocations in dynamic economies with private information. However, it limits the analysis to implementation via direct mechanisms. While such mechanisms can be interpreted as tax systems, they seem divorced from the actual combination of markets and taxes that are used in practice to allocate resources, at least within modern, industrialised economies. ${ }^{4}$ In contrast, we consider fiscal implementations in which agents choose labour supplies and trade claims to future consumption in each period and taxes are conditioned on the agents' observable trades.

Although we analyse fiscal implementations, we do rely on a key insight from the dynamic contracting literature in constructing them. As Green (1987) and others have shown, direct mechanisms are naturally recursive in promised utilities. Similarly, in our fiscal implementations, equilibrium allocations are recursive in an agent's wealth. In the same way that promised utility encodes an agent's history under the direct mechanism, wealth encodes an agent's history in a fiscal implementation. The government is able to infer from an agent's wealth the continuation allocation to which she is entitled. Since the tax system is designed to induce the agent to choose this allocation, it is essential that taxes depend on wealth. Thus, the informational role of wealth is central in determining how it is taxed.

Despite this parallel between direct mechanisms and fiscal implementations, the existence of the former does not imply the existence of the latter. Under a direct mechanism, an agent reports her privately observed shocks to a planner and receives allocations of consumption and labour. By adopting different reporting strategies she can obtain different allocations. In the market economy, an agent chooses budgetfeasible consumption and labour supply allocations. Since the constrained efficient allocation is incentivecompatible, it can be implemented if the set of budget-feasible allocations in the market economy equals the set of allocations available to an agent under the direct mechanism. However, with a simple tax system restricted to condition on current wealth and labour earnings only, the first set is strictly larger than the second. Specifically, an agent in the market economy might choose a labour supply that is consistent with

\footnotetext{
${ }^{2}$ Brito et al. (1991), da Costa and Werning (2001) and Kocherlakota (2003) apply the Mirrlees approach in a dynamic setting. Golosov and Tsyvinski (2003) apply a similar strategy to the analysis of disability insurance.

${ }^{3}$ A selected reference list includes: Green (1987), Phelan \& Townsend (1991), Atkeson \& Lucas (1992, 1995), and Phelan (1994).

${ }^{4}$ They may more closely resemble the arrangements used in simple village economies, see Townsend (1995) or Ligon (1998).
} 
constrained efficient behaviour given a particular history of shocks, but then allocate her after-tax resources between consumption and savings in a way that matches constrained efficient behaviour given a different history of shocks. The tax system cannot verify consistency of the previous period's labour earnings with this period's wealth, nor can it ensure that an agent's savings are consistent with her labour earnings. Surprisingly, when agents' preferences are separable in consumption and labour and when idiosyncratic shocks are i.i.d., we show that it is possible to design a tax system, conditioned on current wealth and labor earnings only, that induces agents to choose the constrained efficient allocation.

The binding incentive-compatibility constraints imply that constrained-efficient allocations satisfy a pattern of wedges between marginal rates of transformation and individual marginal rates of substitution. The optimal tax function is shaped by this pattern. In particular, marginal tax rates must be such that the agent's first order conditions hold at the constrained efficient allocation. However, simply matching marginal taxes to these wedges does not guarantee that the constrained efficient allocation will be implemented. Inducing agents to choose this allocation in the market economy places more structure on marginal tax rates.

Constrained efficient allocations in a dynamic setting are characterised by an intertemporal wedge. A marginal increase in savings at date $t$ exacerbates the incentive problem at $t+1$ since it reduces the correlation between consumption and labor supply in that period. It follows that the cost of a marginal increase in savings is greater than the marginal utility of forgone current consumption, giving rise to a gap between the planner's intertemporal marginal rate of substitution, $q_{t}$, and the agent's expected intertemporal marginal rate of substitution at the constrained-efficient allocation ${ }^{5}$ :

$$
q_{t}<\beta E_{t}\left[\frac{u^{\prime}\left(c_{t+1}\right)}{u^{\prime}\left(c_{t}\right)}\right]
$$

It is natural to suppose that a positive marginal asset tax is needed to implement this wedge, thus giving rise to a rationale for the taxation of assets that is often absent from complete information Ramsey models. However, we show the presence of an intertemporal wedge does not necessarily translate into a positive expected marginal asset tax.

The agent's intertemporal Euler equation in our market economy is of the form:

$$
q_{t} u^{\prime}\left(c_{t}\right)=\beta E_{t}\left[\left(1-\frac{\partial T_{t+1}}{\partial b}\left(b_{t+1}, y_{t+1}\right)\right) u^{\prime}\left(c_{t+1}\right)\right] .
$$

\footnotetext{
${ }^{5}$ This result was initially derived by Diamond and Mirrlees (1978) and Rogerson (1985). Golosov, Kocherlakota and Tsyvinski (2003) provide an extremely general derivation.
} 
Here, $q_{t}$ represents the price at time $t$ of a claim to period $t+1$ consumption, ${ }^{6} T_{t+1}\left(b_{t+1}, y_{t+1}\right)$ denotes taxes in period $t+1$ as a function of an agent's wealth $b_{t+1}$ and labour income $y_{t+1}$, and $\frac{\partial T_{t+1}}{\partial b}$ is the marginal asset tax. An agent's labour income $y_{t+1}$ will vary with the idiosyncratic shock at date $t+1$. Hence, by allowing $\frac{\partial T_{t+1}}{\partial b}$ to depend on $y_{t+1}$ we admit the possibility of a marginal asset tax that is stochastic from the point of view of an agent selecting $b_{t+1}$ at time $t$. The agent's intertemporal Euler equation can be rewritten as:

$$
\beta E_{t}\left[u^{\prime}\left(c_{t+1}\right)\right]-q_{t} u^{\prime}\left(c_{t}\right)=\beta\left\{E_{t}\left[\frac{\partial T_{t+1}}{\partial b}\right] E_{t}\left[u^{\prime}\left(c_{t+1}\right)\right]+\operatorname{Cov}_{t}\left[\frac{\partial T_{t+1}}{\partial b}, u^{\prime}\left(c_{t+1}\right)\right]\right\} \geq 0 .
$$

This decomposition illustrates two ways in which the marginal taxation of wealth can generate an intertemporal wedge. The most direct way is to reduce the expected return on savings by having a positive expected marginal asset tax $E_{t}\left[\frac{\partial T_{t+1}}{\partial b}\right]>0$. The second way is to set marginal asset taxes such that the after-tax return on savings covaries positively with consumption, implying $\operatorname{Cov}_{t}\left[\frac{\partial T_{t+1}}{\partial b}, \frac{u^{\prime}\left(c_{t+1}\right)}{u^{\prime}\left(c_{t}\right)}\right]>0$. This discourages savings in period $t$ by making claims a less effective hedge against period $t+1$ consumption risk. Crucially, (2) shows that there is no presumption that the intertemporal wedge translates into positive expected marginal asset taxes.

We illustrate with an example that a negative covariance between the marginal asset tax and labor income is required to implement the constrained-efficient allocation, in order to rule out "joint deviations" in which agents save too much in period $t$ and work too little in period $t+1$. It follows that the tax function must be non-separable in wealth and current labor income. We then describe how our optimal tax system guarantees that such deviations are sub-optimal for the agent. In addition, we provide an example in which, despite a positive intertemporal wedge, the expected marginal asset tax is zero. We show that, more generally, if the allocation to be implemented at $t+1$ prescribes that labor supply should be lower for agents with higher wealth, the expected marginal asset tax will be positive.

Constrained-efficient allocations in our model also satisfy a lower bound on continuation utilities, as in Atkeson and Lucas (1995). We implement this constraint with a borrowing limit in the market economy. The lower bound on continuation utility implies that, in a neighbourhood of this bound, the constrainedefficient allocation for our dynamic setting will resemble one arising from a static model since there must be a smaller reliance on continuation utility to provide incentives. This will translate into greater curvature of the optimal tax function in a neighbourhood of the borrowing limit in the market economy.

\footnotetext{
${ }^{6}$ This price equals the planner's intertemporal marginal rate of substitution in the direct mechanism.
} 
We explore the steady state properties of the optimal tax system in numerical examples. Our benchmark parameterisation is consistent with recent calibrations of Bewley economies with endogenous labour supply. We find that the optimal tax system displays a strong dependence of marginal asset and marginal income taxes on wealth, as well as a high curvature of optimal tax function in the neighbourhood of the borrowing limit. The optimal marginal labour income tax is high at low wealth and decreasing in wealth. Recent contributions to the static optimal non-linear income taxation literature, such as Diamond (1998) and Saez (2001), find that optimal marginal income taxes are high at low income levels and decreasing in income. This result is interpreted as being consistent with the rapid phasing out of social benefits at low incomes, but is sensitive to assumptions on preferences and the shock distribution. In contrast, the negative dependence of marginal income taxes on wealth in our model is robust to alternative parameterisations. This feature is particularly marked in the neighbourhood of the borrowing limit, where there is a need to provide insurance to agents who are restricted in their ability to smooth consumption intertemporally via asset markets. On the other hand, we also find the dependence of marginal labor income taxes on labour to be sensitive to parameters.

For all our computed examples, the intertemporal wedge is small away from the borrowing limit. It is less than $1 \%$ over most of the wealth range, but close to this limit it rises steeply to a peak of $16 \%$ in our benchmark parameterisation. The marginal asset tax is very close to zero for wealth levels away from the borrowing limit. The expected marginal asset tax is small, it equals approximately $2 \%$ at the borrowing limit and falls steadily with wealth. The covariance between the marginal asset tax and the marginal utility of consumption also falls with wealth, but it is much larger close to the limit and declines steeply as wealth increases. This covariance plays the major role in generating the intertemporal wedge only when this wedge is large. The small computed value of the intertemporal wedge does not imply that taxation of asset income is small in our economy. The equilibrium net rate of return on assets is approximately $10 \%$ in our computed examples, implying that the corresponding marginal tax on asset income is equal to our marginal asset tax augmented by a factor of 10 .

Our paper is closely related to Kocherlakota (2003). He also derives a tax system that implements constrained-optimal allocations in an environment similar to ours. His analysis allows for persistent idiosyncratic shocks. Kocherlakota's tax system is not recursive and does not exploit the information conveyed by an agent's asset position. Instead, it conditions taxes on agents' entire history of labour earnings. Thus, his tax system, while more general, is also much more complex than ours. Interestingly, Kocherlakota's 
optimal tax system always implies a zero expected marginal asset tax. As we discuss in the body of the paper, results on marginal asset taxes are sensitive to the way in which the tax system uses information on the agents' past history.

The remainder of the paper proceeds as follows. In section 2, we state the planner's problem for economies of arbitrary finite length. We provide a recursive formulation for the planner's problem that is closely related to those in Atkeson and Lucas $(1992,1995)$. In section 3, we prove our main implementation result for an economy with two periods and then extend the proof to economies of arbitrary finite length. Section 4 studies the optimal pattern of wedges that characterise constrained-efficient allocations. In this section we begin to discuss the relationship between wedges and taxes in a dynamic setting. Section 5 provides a series of examples that analyze this relationship in a two-period setting. In section 6 , we extend our implementation results to economies of infinite length. We present a numerical analysis of the optimal tax system in the steady state of an infinite period economy in section 7 . In this section, we also compare our optimal tax system with the findings of the static optimal non-linear income taxation literature. Section 8 concludes.

\section{The planner's problem in a finite period economy}

In this section, we formally describe the planner's problem in a finite period economy. The economy is inhabited by a continuum of agents. These agents have preferences over stochastic sequences $\left\{c_{t}, y_{t}\right\}_{t=0}^{T}$ of consumption $c_{t} \in \mathbb{R}_{+}$and labour $y_{t} \in \mathcal{Y}: \equiv[0, \bar{y}]$ of the form:

$$
E\left[\sum_{t=0}^{T} \beta^{t}\left[u\left(c_{t}\right)+\theta_{t} v\left(y_{t}\right)\right]\right] .
$$

We assume that $u: \mathbb{R}_{+} \rightarrow \mathcal{U} \subset \mathbb{R}$ and $v: \mathcal{Y} \rightarrow \mathcal{V} \subset \mathbb{R}$ are continuously differentiable, strictly concave and, respectively, strictly increasing and strictly decreasing functions. The variable $\theta_{t} \in \Theta \subset \mathbb{R}$ denotes an idiosyncratic preference shock. We assume that $\Theta$ is a compact set and that the preference shocks are distributed independently over time and across agents with probability distribution $\pi .^{7}$ We define a $t$-period history to be $\theta^{t}=\left(\theta_{0}, \ldots, \theta_{t}\right) \in \Theta^{t+1}$ and denote the corresponding probability distribution by

\footnotetext{
${ }^{7}$ The distribution function $\pi$ also describes the cross sectional distribution of $\theta_{t}$ at each $t$, which amounts to assuming that the law of large numbers holds across agents. As is well known, the law of large numbers may not apply if the underlying index space is a Borel measure. We implicitly rely on the construction of Judd (1985) to resolve this issue.
} 
$\pi^{t}$. We assume that the idiosyncratic shocks are privately observed by agents. The term $\theta_{t} v\left(y_{t}\right)$ denotes the disutility from labour at time $t$. Preference shocks alter the disutility of labour and the marginal rate of substitution between consumption and labour. They may, for example, be interpreted as short-lived shocks to health. We will focus on this interpretation and also assume that the production technology converts one unit of labour into one unit of output. The preference shock formulation that we adopt can easily be mapped into one in which agents receive privately observed productivity shocks that perturb their individual marginal rates of transformation of labour into output.

Each agent is identified with a number $w_{0}$ which represents the agent's initial entitlement to expected lifetime utility, or initial utility promise. Let $\Psi_{0}$ denote the distribution over initial promises and let $\mathcal{W}_{0} \subseteq \mathbb{R}$ denote its support. It is convenient to state the planner's problem in terms of utility, rather than resource, variables. Given $\mathcal{W}_{0}$, define a utility allocation to be a sequence of functions $z=\left\{u_{t}, v_{t}\right\}_{t=0}^{T}$ with $u_{t}: \mathcal{W}_{0} \times \Theta^{t+1} \rightarrow \mathcal{U}$ and $v_{t}: \mathcal{W}_{0} \times \Theta^{t+1} \rightarrow \mathcal{V}$. Here $u_{t}$ and $v_{t}$ give the utility obtained by an agent from consumption and labour at date $t$ as a function of that agent's utility promise and shock history. An individual utility allocation for an agent with initial promise $w_{0}$ will be denoted $z\left(w_{0}\right)^{8}$. Let $C: \mathcal{U} \rightarrow \mathbb{R}_{+}$ denote the inverse of $u$ and $Y: \mathcal{V} \rightarrow \mathcal{Y}$ the inverse of $v$. An individual utility allocation can be mapped into a consumption-labour allocation using the functions $C$ and $Y$. Denote an agent's continuation utility from the individual utility allocation $z\left(w_{0}\right)$ at date $s$ by:

$$
U_{s}\left(z\left(w_{0}\right), \theta^{s}\right)=E\left[\sum_{t=s}^{T} \beta^{t-s}\left[u_{t}\left(w_{0}, \theta^{t}\right)+\theta_{t} v_{t}\left(w_{0}, \theta^{t}\right)\right] \mid \theta^{s}\right] \quad s=0, \ldots, T
$$

with $U_{T+1}\left(z\left(w_{0}\right), \theta^{T+1}\right) \equiv 0$. Let $\bar{U}_{s}^{\text {nat }}: \equiv \sup _{\varphi \in \mathcal{U}, \varsigma \in \mathcal{V}} \frac{1-\beta^{T+1-s}}{1-\beta}[\varphi+E \theta \varsigma]$ denote an upper bound for $U_{s}$.

Having introduced notation, we now state the constraints imposed on the planner's problem. First we require that the planner delivers $w_{0}$ to those entitled to $w_{0}$. Formally, the planner must choose a utility allocation $z$ that satisfies the promise keeping condition, for all $w_{0} \in \mathcal{W}_{0}{ }^{9}$

$$
w_{0}=U_{0}\left(z\left(w_{0}\right)\right)
$$

Second since agents privately observe their histories of shocks, we require that the planner selects an incentive-compatible utility allocation. We define a reporting strategy $\delta$ to be a sequence of functions

\footnotetext{
${ }^{8}$ The strict concavity of the problem ensures that it is optimal for a planner to treat all agents with the same utility promise identically. Hence, there is no loss of generality in assuming that agents with the same promise receive the same allocation.

${ }^{9}$ We will assume throughout that for each $w_{0} \in \mathcal{W}_{0}$, there exists a utility allocation $z$ such that $w_{0}=U_{0}(z)$.
} 
$\left\{\delta_{t}\right\}_{t=0}^{T}$ with $\delta_{t}: \Theta^{t} \rightarrow \Theta$. We interpret $\delta_{t}$ as mapping an agent's history of shocks into a report concerning her current shock. Let $z\left(w_{0} ; \delta\right)$ denote the composition of the individual utility allocation $z\left(w_{0}\right)$ and the reporting strategy $\delta$. This is also an individual utility allocation. Let $\delta^{*}=\left\{\delta_{t}^{*}\right\}_{t=0}^{T}$ denote the truthful reporting strategy, where for all $t, \theta^{t}, \delta_{t}^{*}\left(\theta^{t-1}, \theta_{t}\right)=\theta_{t}$. We invoke the Revelation Principle and, without loss of generality, require that utility allocations induce agents to be truthful ${ }^{10}$. Thus, we restrict the planner to choose utility allocations $z$ that satisfy the incentive compatibility condition, for all $w_{0} \in \mathcal{W}_{0}$

$$
\forall \delta: U_{0}\left(z\left(w_{0} ; \delta^{*}\right)\right) \geq U_{0}\left(z\left(w_{0} ; \delta\right)\right)
$$

We will say that a utility allocation $z=\left\{u_{t}, v_{t}\right\}_{t=0}^{T}$ is temporarily incentive-compatible if for all $w_{0}$

$$
\begin{aligned}
& \forall t, \theta^{t-1}, \theta, \theta^{\prime}, \quad u_{t}\left(w_{0}, \theta^{t-1}, \theta\right)+\theta v_{t}\left(w_{0}, \theta^{t-1}, \theta\right)+\beta U_{t+1}\left(z\left(w_{0}\right), \theta^{t-1}, \theta\right) \\
& \geq u_{t}\left(w_{0}, \theta^{t-1}, \theta^{\prime}\right)+\theta v_{t}\left(w_{0}, \theta^{t-1}, \theta^{\prime}\right)+\beta U_{t+1}\left(z\left(w_{0}\right), \theta^{t-1}, \theta^{\prime}\right) .
\end{aligned}
$$

The latter constraints imply that after each history of shocks, an agent is better off truthfully reporting her shock, rather than lying and being truthful thereafter. (5) clearly implies (6) and, in a finite period setting, the reverse implication is also true. We will require that the planner chooses utility allocations satisfying the more tractable constraints in (6).

Finally, the planner will be mandated to keep the continuation utilities of agents above an exogenous lower bound, $\underline{U}_{t+1}<\bar{U}_{t+1}^{n a t}$ at each date. Thus, utility allocations must satisfy, for all $w_{0} \in \mathcal{W}_{0}$,

$$
\forall t \in\{0, \ldots, T-1\}, \theta^{t}, \quad U_{t+1}\left(z\left(w_{0}\right) \mid \theta^{t}\right) \geq \underline{U}_{t+1} .
$$

Define $\mathcal{W}_{t}=\left[\underline{U}_{t+1}, \infty\right) \cap \operatorname{Range}\left(U_{t}\right)$ to be the set of possible expected period $t$ payoffs for an agent, and let $\left\{G_{t}\right\}_{t=0}^{T}$ denote a sequence of exogenous planner consumption levels. The planner's cost objective is given by:

$$
D\left(z ;\left\{G_{t}\right\}_{t=0}^{T}, \Psi_{0}\right)=\max _{t \in\{0, \ldots, T\}}\left\{\int_{w_{0} \in \mathcal{W}_{0}} \int_{\theta^{t} \in \Theta^{t+1}}\left[C\left(u_{t}\left(w_{0}, \theta^{t}\right)\right)-Y\left(v_{t}\left(w_{0}, \theta^{t}\right)\right)\right] d \pi^{t} d \Psi_{0}+G_{t}\right\} .
$$

The planner minimises this cost objective subject to the promise keeping, incentive-compatibility and utility bound constraints:

$$
\begin{aligned}
\mathcal{C}\left(\left\{\underline{U}_{t+1}\right\}_{t=0}^{T-1},\left\{G_{t}\right\}_{t=0}^{T}, \Psi_{0}\right)= & \inf _{z} D\left(z ;\left\{G_{t}\right\}_{t=0}^{T}, \Psi_{0}\right) \\
\text { subject to: } & \forall w_{0},(4),(6) \text { and }(7) .
\end{aligned}
$$

\footnotetext{
${ }^{10}$ We assume throughout that the planner can commit to implementing a particular report contingent utility allocation.
} 
If $z^{*}=\left\{u_{t}^{*}, v_{t}^{*}\right\}_{t=0}^{T}$ attains the infimum in (9) then we will call $z^{*}$ a constrained efficient allocation (at $\left.\left(\left\{\underline{U}_{t+1}\right\}_{t=0}^{T-1},\left\{G_{t}\right\}_{t=0}^{T}, \Psi_{0}\right)\right)$. We say that a triple $\left(\left\{\underline{U}_{t+1}\right\}_{t=0}^{T-1},\left\{G_{t}\right\}_{t=0}^{T}, \Psi_{0}\right)$ is consistent with resource clearing if there exists a constrained efficient allocation at $\left(\left\{\underline{U}_{t+1}\right\}_{t=0}^{T-1},\left\{G_{t}\right\}_{t=0}^{T}, \Psi_{0}\right)$ that satisfies, for $t \in\{0, \ldots T\}$,

$$
\int_{w_{0} \in \mathcal{W}_{0}} \int_{\theta^{t} \in \Theta^{t+1}}\left[C\left(u_{t}\left(w_{0}, \theta^{t}\right)\right)-Y\left(v_{t}\left(w_{0}, \theta^{t}\right)\right)\right] d \pi^{t} d \Psi_{0}+G_{t}=0 .
$$

We now briefly summarise some well known results about problem (9). In this problem, a single planner allocates resources across a population of agents. Following Atkeson and Lucas (1992), (9) can be formulated as a collection of component planning problems. In each of these a component planner is responsible for allocating resources only to those agents with a specific initial utility promise of $w_{0}$. Each component planner delivers $w_{0}$, respecting incentive compatibility and the lower bound on continuation utilities, at minimal cost, where cost is computed using a sequence of intertemporal prices $\left\{q_{t}\right\}_{t=0}^{T-1} \in \mathbb{R}_{+}^{T} \cdot q_{t}$ may be interpreted as the social shadow price of period $t+1$ consumption in terms of period $t$ consumption. ${ }^{11}$ Formally, the component planners solve:

$$
B_{0}\left(w_{0}\right)=\inf _{\left\{u_{t}^{\prime}, v_{t}^{\prime}\right\}_{t=0}^{T}} \sum_{t=0}^{T} Q_{t} \int_{\theta^{t} \in \Theta^{t+1}}\left[C\left(u_{t}^{\prime}\left(\theta^{t}\right)\right)-Y\left(v_{t}^{\prime}\left(\theta^{t}\right)\right)\right] d \pi^{t}
$$

$$
\text { subject to : (4), (6) and (7), }
$$

where $Q_{0}=1$ and, for $t \geq 1, Q_{t}: \equiv \prod_{s=0}^{t-1} q_{t}$. These planners can be thought of as trading claims to future consumption amongst themselves at the price $q_{t}$ in period $t$. Lemma 1 links this component planning formulation to problem (9).

Lemma 1 Fix $\left(\left\{\underline{U}_{t+1}\right\}_{t=0}^{T-1},\left\{G_{t}\right\}_{t=0}^{T}, \Psi_{0}\right)$. Suppose there exists a utility allocation $z$ and a sequence of intertemporal prices $\left\{q_{t}\right\}_{t=0}^{T-1}$ such that:

$$
\text { 1. for all } t, \int_{w_{0} \in \mathcal{W}_{0}} \int_{\theta^{t} \in \Theta^{t+1}}\left[C\left(u_{t}\left(w_{0}, \theta^{t}\right)\right)-Y\left(v_{t}\left(w_{0}, \theta^{t}\right)\right)\right] d \pi^{t} d \Psi_{0}+G_{t}=0 \text { and }
$$

\footnotetext{
${ }^{11}$ In Albanesi and Sleet (2004) we show that if $\Psi_{0}$ is consistent with resource clearing, then the associated constrained efficient allocation also solves a "primal problem" in which the planner maximises a Pareto-weighted aggregate of agent utilities subject to a resource constraint, incentive-compatibility and utility bound constraints. Conversely, any allocation that solves such a primal problem is also the solution to a dual problem (9) with an appropriately set utility promise distribution $\Psi_{0}$. Moreover, this $\Psi_{0}$ is consistent with resource clearing. In the primal problem $q_{t}$ is the shadow price of consumption at date $t+1$ in terms of consumption at date $t$.
} 
2. for all $w_{0}, z\left(w_{0}\right)$ solves the corresponding component planning problem (10).

Then, $z$ is constrained efficient at $\left(\left\{\underline{U}_{t+1}\right\}_{t=0}^{T-1},\left\{G_{t}\right\}_{t=0}^{T}, \Psi_{0}\right)$ and $\left(\left\{\underline{U}_{t+1}\right\}_{t=0}^{T-1},\left\{G_{t}\right\}_{t=0}^{T}, \Psi_{0}\right)$ is consistent with resource clearing.

Proof: See Atkeson and Lucas (1992).

The component planning problem (10) is recursive in the agent's utility promise. We reformulate it to take advantage of this recursivity. We then re-express Conditions 1 and 2 of Lemma 1 as equilibrium conditions for a recursive component planner economy. This recursive formulation is a convenient first step towards obtaining our main implementation result.

Fix a triple $\left(\left\{\underline{U}_{t+1}\right\}_{t=0}^{T-1},\left\{G_{t}\right\}_{t=0}^{T}, \Psi_{0}\right)$ and, hence, the sequence $\left\{\mathcal{W}_{t}\right\}_{t=0}^{T}$. Define a utility allocation rule to be a collection of functions $\zeta=\left\{\left\{\varphi_{t}, \varsigma_{t}, \omega_{t+1}\right\}_{t=0}^{T-1}, \varphi_{T}, \varsigma_{T}\right\}$ with $\varphi_{t}: \mathcal{W}_{t} \times \Theta \rightarrow \mathcal{U}, \varsigma_{t}: \mathcal{W}_{t} \times \Theta \rightarrow \mathcal{V}$ and $\omega_{t+1}: \mathcal{W}_{t} \times \Theta \rightarrow \mathcal{W}_{t+1}$. The functions $\left.\varphi_{t}\left(w_{t}, \theta_{t}\right), \varsigma_{t}\left(w_{t}, \theta_{t}\right)\right)$ and $\omega_{t+1}\left(w_{t}, \theta_{t}\right)$ represent, respectively, the utility from current consumption, labour supply, and the period $t+1$ utility promise assigned to an agent with current utility promise $w_{t}$ and shock $\theta_{t}$. A utility allocation rule recursively induces a utility allocation as follows. Given $\zeta$ and an initial utility promise $w_{0} \in \mathcal{W}_{0}$, define $w_{t+1}\left(w_{0}, \theta^{t}\right)=\omega_{t+1}\left(w_{t}\left(w_{0}, \theta^{t-1}\right), \theta_{t}\right)$, then for all $t, w_{0}$ and $\theta^{t-1}$ set $u_{t}\left(w_{0}, \theta^{t}\right)=\varphi_{t}\left(w_{t}\left(w_{0}, \theta^{t-1}\right), \theta_{t}\right)$ and $v_{t}\left(w_{0}, \theta^{t}\right)=\varsigma_{t}\left(w_{t}\left(w_{0}, \theta^{t-1}\right), \theta_{t}\right)$. Thus, the utility allocation rule uses utility promises to summarise past information. We denote with $z\left(\zeta, w_{0}\right)$ the individual utility allocation induced by $\zeta$ from $w_{0}$.

In the recursive formulation of (10), the component planner's problem in period $t \in\{0, \ldots, T-1\}$ is:

$$
B_{t}\left(w_{t}\right)=\inf _{\substack{\varphi: \Theta \rightarrow \mathcal{U}, \varsigma: \Theta \rightarrow \mathcal{V} \\ \omega: \Theta \rightarrow \mathcal{W}_{t+1}}} \int_{\Theta}\left[C(\varphi(\theta))-Y(\varsigma(\theta))+q_{t} B_{t+1}(\omega(\theta))\right] d \pi
$$

subject to the temporary incentive-compatibility constraint:

$$
\forall \theta, \theta^{\prime}: \varphi(\theta)+\theta \varsigma(\theta)+\beta \omega(\theta) \geq \varphi\left(\theta^{\prime}\right)+\theta \varsigma\left(\theta^{\prime}\right)+\beta \omega\left(\theta^{\prime}\right)
$$

and the promise-keeping constraint:

$$
w_{t}=\int_{\Theta}[\varphi(\theta)+\theta \varsigma(\theta)+\beta \omega(\theta)] d \pi
$$

In the terminal period $T$, the component planner solves:

$$
B_{T}\left(w_{T}\right)=\inf _{\substack{\varphi: \Theta \rightarrow \mathcal{U} \\ \varsigma: \Theta \rightarrow \mathcal{V}}} \int_{\Theta}[C(\varphi(\theta))-Y(\varsigma(\theta))] d \pi
$$


subject to the temporary incentive-compatibility constraint:

$$
\forall \theta, \theta^{\prime}: \varphi(\theta)+\theta \varsigma(\theta) \geq \varphi\left(\theta^{\prime}\right)+\theta \varsigma\left(\theta^{\prime}\right)
$$

and the promise-keeping constraint:

$$
w_{T}=\int_{\Theta}[\varphi(\theta)+\theta \varsigma(\theta)] d \pi .
$$

The solution to problems (11) and (14) defines a utility allocation rule, $\zeta^{*}$. By a standard argument $z\left(\zeta^{*}, w_{0}\right)$ attains the infimum in (10). The following pair of lemmas provide some basic characterisation of this sequence of value functions $\left\{B_{t}\right\}_{t=0}^{T}$ and of $\zeta^{*}$.

Lemma 2 Each $B_{t}, t \in\{0, \ldots, T\}$, is increasing and strictly convex.

Proof: See Appendix.

Lemma 3 1. There exists a utility allocation rule $\zeta^{*}=\left\{\left\{\varphi_{t}^{*}, \varsigma_{t}^{*}, \omega_{t+1}^{*}\right\}_{t=0}^{T-1}, \varphi_{T}^{*}, \varsigma_{T}^{*}\right\}$ that solves the recursive component planner's problem. Specifically, for each $t, w_{t} \in \mathcal{W}_{t}$, the functions $\left\{\varphi_{t}^{*}\left(w_{t}, \cdot\right), \varsigma_{t}^{*}\left(w_{t}, \cdot\right)\right.$, $\left.\omega_{t+1}^{*}\left(w_{t}, \cdot\right)\right\}$ attain the infima in (11); for each $w_{T} \in \mathcal{W}_{T}$, the functions $\left\{\varphi_{T}^{*}\left(w_{T}, \cdot\right), \varsigma_{T}^{*}\left(w_{T}, \cdot\right)\right\}$ attain the infima in (14).

2. Each function $\varphi_{t}^{*}\left(w_{t}, \cdot\right), \varsigma_{t}^{*}\left(w_{t}, \cdot\right), \omega_{t+1}^{*}\left(w_{t}, \cdot\right)$ is monotone.

3. For each $t$, there exist functions $\left\{c_{t}^{*}, y_{t}^{*}, B_{t+1}^{*}\right\}$ such that for all $w \in \mathcal{W}_{t}$ and $\Theta$,

$$
\begin{aligned}
y_{t}^{*}\left(B_{t}(w), \theta\right) & =Y\left(\varsigma_{t}^{*}(w, \theta)\right) \\
c_{t}^{*}\left(B_{t}(w), \theta\right) & =C\left(\varphi_{t}^{*}(w, \theta)\right) \\
B_{t+1}^{*}\left(B_{t}(w), \theta\right) & =B_{t+1}\left(\omega_{t+1}^{*}(w, \theta)\right) .
\end{aligned}
$$

Proof: See Appendix.

The final part of Lemma 3 re-expresses the optimal labour supply, consumption and component planner's continuation cost, in the recursive component planner problem, as functions of the component planner's cost and the shock. We will call the sequence $\alpha^{*}=\left\{\left\{y_{t}^{*}, c_{t}^{*}, B_{t+1}^{*}\right\}_{t=0}^{T-1}, y_{T}^{*}, c_{T}^{*}\right\}$ a resource allocation rule. It uses planner costs, rather than utility promises, to summarise the past. We also define $\mathcal{Y}_{t}^{*}: B_{t}\left(\mathcal{W}_{t}\right) \rightrightarrows \mathcal{Y}$ by

$$
\mathcal{Y}_{t}^{*}(b): \equiv\left\{y: y=y_{t}^{*}(b, \widetilde{\theta}) \text { some } \tilde{\theta} \in \Theta\right\} \subseteq \mathcal{Y}
$$


$\mathcal{Y}_{t}^{*}(b)$ is the set of labour supplies available to an agent with utility promise $B_{t}^{-1}(b)$ at date $t$ under $\zeta^{*}$.

The optimal promise functions $\left\{\omega_{t+1}^{*}\right\}_{t=0}^{T-1}$ and the distribution $\Psi_{0}$ induce a sequence of cross sectional utility promise distributions $\Psi_{t+1}$ according to:

$$
\forall S \in \mathbf{B}\left(\mathcal{W}_{t+1}\right): \quad \Psi_{t+1}(S)=\int 1_{\left\{\omega_{t+1}^{*}(w, \theta) \in S\right\}} d \pi d \Psi_{t}
$$

where $\mathbf{B}\left(\mathcal{W}_{t+1}\right)$ denotes the Borel subsets of $\mathcal{W}_{t+1}$. Additionally, $\zeta^{*}$ and $\left\{\Psi_{t}\right\}_{t=0}^{T}$ imply a sequence of aggregate resource costs for $t=0, \ldots, T$ :

$$
\int_{\mathcal{W}_{t}} \int_{\Theta}\left[C\left(\varphi_{t}^{*}(w, \theta)\right)-Y\left(\varsigma_{t}^{*}(w, \theta)\right)\right] d \pi d \Psi_{t}+G_{t} .
$$

Define a component planner economy, denoted $\mathcal{E}^{C P}\left(\left\{\underline{U}_{t+1}\right\}_{t=0}^{T-1},\left\{G_{t}\right\}_{t=0}^{T}, \Psi_{0}\right)$, to be a continuum of component planners, an initial cross sectional distribution of utility promises $\Psi_{0}$, a sequence of continuation utility bounds $\left\{\underline{U}_{t+1}\right\}_{t=0}^{T-1}$, a sequence of markets for one period ahead claims to consumption between periods 0 and $T-1$, and a sequence of government consumptions $\left\{G_{t}\right\}_{t=0}^{T}$. We use our recursive formulation of the component planning problems to define an equilibrium for this economy.

Definition 1 A sequence of intertemporal prices $\left\{q_{t}\right\}_{t=0}^{T-1}$, a utility allocation rule $\zeta^{*}=\left\{\left\{\varphi_{t}^{*}, \varsigma_{t}^{*}, \omega_{t+1}^{*}\right\}_{t=0}^{T-1}\right.$, $\left.\varphi_{T}^{*}, \varsigma_{T}^{*}\right\}$, cost functions $\left\{B_{t}\right\}_{t=0}^{T}$, with $B_{t}: \mathcal{W}_{t} \rightarrow \mathbb{R}$, and a sequence of cross sectional distributions of utility promises $\left\{\Psi_{t}\right\}_{t=1}^{T}$ are an equilibrium of $\mathcal{E}^{C P}\left(\left\{\underline{U}_{t+1}\right\}_{t=0}^{T-1},\left\{G_{t}\right\}_{t=0}^{T}, \Psi_{0}\right)$ if:

1. $\left\{B_{t}\right\}_{t=0}^{T-1}$ satisfy (11) and $B_{T}$ satisfies (14);

2. $\left\{\varphi_{t}^{*}, \varsigma_{t}^{*}, \omega_{t+1}^{*}\right\}$ attain the infima in the problems (11). $\left\{\varphi_{T}^{*}, \varsigma_{T}^{*}\right\}$ attain the infima in the problems (14);

3. $\forall t, S \in \mathbf{B}\left(\mathcal{W}_{t+1}\right), \Psi_{t+1}(S)=\int 1_{\left\{\omega_{t+1}^{*}(w, \theta) \in S\right\}} d \pi d \Psi_{t}$

4. $\forall t, G_{t}+\int\left[C\left(\varphi_{t}^{*}(w, \theta)\right)-Y\left(\varsigma_{t}^{*}(w, \theta)\right)\right] d \pi d \Psi_{t}=0$.

Conditions 1 to 4 of this definition restate the conditions of Lemma 1 in terms of a recursive component planner economy. It follows that if $\zeta^{*}$ is an equilibrium utility allocation rule for a component planner economy $\mathcal{E}^{C P}\left(\left\{\underline{U}_{t+1}\right\}_{t=0}^{T-1},\left\{G_{t}\right\}_{t=0}^{T}, \Psi_{0}\right)$ then the utility allocation that it induces is constrained efficient at $\left(\left\{\underline{U}_{t+1}\right\}_{t=0}^{T-1},\left\{G_{t}\right\}_{t=0}^{T}, \Psi_{0}\right)$. We will call such a $\zeta^{*}$ a constrained efficient utility allocation rule. By Lemma 3 , a constrained efficient utility allocation rule $\zeta^{*}$ can be equivalently expressed as a resource allocation rule $\alpha^{*}$. We will call the latter a constrained efficient resource allocation rule. 


\section{Implementation}

We now show that a component planner equilibrium, and, hence, the associated constrained efficient allocation, can be obtained as part of a competitive equilibrium in a market economy with taxes and borrowing constraints. Agents are endowed with an initial stock of non-contingent claims $b_{0}$. They enter each period $t$ with claims $b_{t}$, they work $y_{t}$, pay taxes and, in periods $t \leq T-1$, they allocate their after-tax income between consumption $c_{t}$ and purchases of claims $b_{t+1}$. In the terminal period $T$, they simply consume all after-tax income. All market trades undertaken by an agent are publicly observable. A government is exogenously assigned the sequence of spending levels $\left\{G_{t}\right\}_{t=0}^{T}$ and administers the tax system, $\left\{T_{t}\right\}_{t=0}^{T}$, that supplements this trading arrangement. The tax system conditions an agent's tax payment in each period only on her current labour income $y_{t}$ and her current stock of claims $b_{t}$ and not on any other aspect of her past history. Thus, it is simple. We show that such a simple tax system implements the constrained-efficient allocation.

Formally, a market economy with taxes and borrowing limits, denoted $\mathcal{E}^{M E}\left(\left\{\underline{b}_{t+1}\right\}_{t=0}^{T-1}, \Lambda_{0},\left\{G_{t}\right\}_{t=0}^{T}\right.$, $\left.\left\{T_{t}\right\}_{t=0}^{T}\right)$, is a sequence of markets for one period ahead claims to consumption that open at each date $t \leq T-1$, a sequence of borrowing limits $\left\{\underline{b}_{t+1}\right\}_{t=0}^{T-1}$, an initial cross sectional distribution of claim holdings $\Lambda_{0}$, a sequence of government spending levels $\left\{G_{t}\right\}_{t=0}^{T}$, and a sequence of tax functions $\left\{T_{t}\right\}_{t=0}^{T}$, with $T_{t}: \mathcal{B}_{t} \times \mathcal{Y} \rightarrow \mathbb{R}$, where for $t>0, \mathcal{B}_{t} \equiv\left[\underline{b}_{t}, \infty\right)$ and $\mathcal{B}_{0}$ denotes the support of $\Lambda_{0}$. We define a market allocation rule to be a sequence of functions $\widehat{a}=\left\{\left\{\widehat{c}_{t}, \widehat{y}_{t}, \widehat{b}_{t+1}\right\}_{t=0}^{T-1}, \widehat{c}_{T}, \widehat{y}_{T}\right\}$, with $\widehat{c}_{t}: \mathcal{B}_{t} \times \Theta \rightarrow \mathbb{R}_{+}$, $\widehat{y}_{t}: \mathcal{B}_{t} \times \Theta \rightarrow \mathcal{Y}$, and $\widehat{b}_{t+1}: \mathcal{B}_{t} \times \Theta \rightarrow \mathcal{B}_{t+1}$. The functions $\widehat{c}_{t}\left(b_{t}, \theta_{t}\right), \widehat{y}_{t}\left(b_{t}, \theta_{t}\right)$ and $\widehat{b}_{t+1}\left(b_{t}, \theta_{t}\right)$ represent, respectively, consumption, labour supply, and savings at time $t$ of an agent with current wealth $\widehat{b}_{t}$ and shock $\theta_{t}$. A competitive equilibrium of the market economy $\mathcal{E}^{M E}\left(\left\{\underline{b}_{t+1}\right\}_{t=0}^{T-1}, \Lambda_{0},\left\{G_{t}\right\}_{t=0}^{T},\left\{T_{t}\right\}_{t=0}^{T}\right)$ is defined as follows.

Definition 2 A sequence of claims prices $\left\{\widehat{q}_{t}\right\}_{t=0}^{T-1} \in \mathbb{R}_{+}^{T}$, a market allocation rule $\widehat{a}=\left\{\left\{\widehat{c}_{t}, \widehat{y}_{t}, \widehat{b}_{t+1}\right\}_{t=0}^{T-1}\right.$, $\left.\widehat{c}_{T}, \widehat{y}_{T}\right\}$, value functions $\left\{V_{t}\right\}_{t=0}^{T}$, with $V_{t}: \mathcal{B}_{t} \rightarrow \mathbb{R}$, and a sequence of cross sectional distributions of claim holdings $\left\{\Lambda_{t+1}\right\}_{t=0}^{T-1}$ is a competitive equilibrium of the market economy with taxes and borrowing limits $\mathcal{E}^{M E}\left(\left\{\underline{b}_{t+1}\right\}_{t=0}^{T-1}, \Lambda_{0},\left\{G_{t}\right\}_{t=0}^{T},\left\{T_{t}\right\}_{t=0}^{T}\right)$ if:

1. for $t \in\{0, \ldots, T-1\}, V_{t}$ and $V_{t+1}$ satisfy:

$$
V_{t}(b)=\sup _{\substack{c: \Theta \rightarrow \mathbb{R}_{+}, y: \Theta \rightarrow \mathcal{Y} \\ b^{\prime}: \Theta \rightarrow \mathcal{B}_{t+1}}} \int\left[u(c(\theta))+\theta v(y(\theta))+\beta V_{t+1}\left(b^{\prime}(\theta)\right)\right] d \pi
$$


subject to, for each $\theta, b=c(\theta)-y(\theta)+T_{t}(b, y(\theta))+\widehat{q}_{t} b^{\prime}(\theta) ; V_{T}$ satisfies

$$
V_{T}(b)=\sup _{c: \Theta \rightarrow \mathbb{R}_{+}, y: \Theta \rightarrow \mathcal{Y}} \int[u(c(\theta))+\theta v(y(\theta))] d \pi
$$

subject to, for each $\theta, b=c(\theta)-y(\theta)+T_{T}(b, y(\theta))$;

2. $\left\{\widehat{c}_{t}, \widehat{y}_{t}, \widehat{b}_{t+1}\right\}$ attain the suprema in the problems (19). $\left\{\widehat{c}_{T}, \widehat{y}_{T}\right\}$ attain the suprema in (20);

3. $\forall S \in \mathbf{B}\left(\mathcal{B}_{t+1}\right), \Lambda_{t+1}(S)=\int 1_{\left\{\widehat{b}_{t+1}(b, \theta) \in S\right\}} d \pi d \Lambda_{t}$

4. $\forall t, G_{t}+\int\left[\widehat{c}_{t}(b, \theta)-\widehat{y}_{t}(b, \theta)\right] d \pi d \Lambda_{t}=0$.

Given an initial wealth $b_{0}$, an equilibrium market allocation rule induces a utility allocation $\hat{z}\left(\hat{a}, b_{0}\right)$ from $b_{0}$. We formally define an implementation as follows.

Definition 3 Let $z^{*}$ be a constrained efficient utility allocation at $\left(\left\{\underline{U}_{t+1}\right\}_{t=0}^{T-1}, \Psi_{0},\left\{G_{t}\right\}_{t=0}^{T}\right)$. We say that $z^{*}$ is implemented by a competitive equilibrium in a market economy with taxes and borrowing limits $\mathcal{E}^{M E}\left(\left\{\underline{b}_{t+1}\right\}_{t=0}^{T-1}, \Lambda_{0},\left\{G_{t}\right\}_{t=0}^{T},\left\{T_{t}\right\}_{t=0}^{T}\right)$ if:

1. there exists a measurable function $f: \mathcal{W}_{0} \rightarrow \mathbb{R}$ such that for each $S \in \mathbf{B}(\mathbb{R}), \Lambda_{0}(S)=\Psi_{0}\left(f^{-1}(S)\right)$;

2. $\mathcal{E}^{M E}\left(\left\{\underline{b}_{t+1}\right\}_{t=0}^{T-1}, \Lambda_{0},\left\{G_{t}\right\}_{t=0}^{T},\left\{T_{t}\right\}_{t=0}^{T}\right)$ has a competitive equilibrium $\xi^{M E}=\left\{\left\{q_{t}\right\}_{t=0}^{T-1}, \widehat{a},\left\{V_{t}\right\}_{t=0}^{T}\right.$, $\left.\left\{\Lambda_{t+1}\right\}_{t=0}^{T-1}\right\}$ such that for each $w_{0} \in \mathcal{W}_{0}, \hat{z}\left(\widehat{a}, f\left(w_{0}\right)\right)=z^{*}\left(w_{0}\right)$.

If $z^{*}$ can be implemented by a competitive equilibrium $\xi^{M E}$ in a market economy $\mathcal{E}^{M E}\left(\left\{\underline{b}_{t+1}\right\}_{t=0}^{T-1}, \Lambda_{0}\right.$, $\left.\left\{G_{t}\right\}_{t=0}^{T},\left\{T_{t}\right\}_{t=0}^{T}\right)$, then $\left(\mathcal{E}^{M E}\left(\left\{\underline{b}_{t+1}\right\}_{t=0}^{T-1}, \Lambda_{0},\left\{G_{t}\right\}_{t=0}^{T},\left\{T_{t}\right\}_{t=0}^{T}\right), \xi^{M E}\right)$ is said to be a fiscal implementation of $z^{*}$.

The first condition in the definition describes how the initial wealth distribution is set in the market economy. It requires that an agent's initial claims in this economy be measurable with respect to the agent's initial utility promise in the planner's problem. Intuitively, measurability implies that initial claim holdings will reveal the agent's initial utility promise to the government. The second condition is the central one. It requires that if an agent is given initial claim holdings of $f\left(w_{0}\right)$ in the market economy, then she will choose the constrained efficient individual utility allocation $z^{*}\left(w_{0}\right)$. 


\subsection{Implementation in a two period economy}

We now show that constrained efficient utility allocations that are induced by the equilibria of component planner economies can be implemented in market economies with taxes and borrowing limits. Although our fiscal implementation result applies to economies of arbitrary finite length, the key insights are most easily seen in a two period setting, and we will initially focus on this case. Subsequently, we extend our results to time horizons $T>1$. Our approach is constructive. Given a component planner economy equilibrium, we propose an initial distribution of claims and a candidate equilibrium claims price for the market economy. We then derive a tax function and debt limits under which agents will be able to afford the constrained efficient allocation from the component planner economy. The challenge will then lie in showing that the agents in the market economy do in fact choose this allocation.

Formally, let $\xi^{C P}=\left\{q, \zeta^{*},\left\{B_{t}\right\}_{t=0}^{1}, \Psi_{1}\right\}$ denote the equilibrium of a two period component planner economy $\mathcal{E}^{C P}\left(\underline{U},\left\{G_{t}\right\}_{t=0}^{1}, \Psi_{0}\right)$. The functions that comprise the utility allocation rule $\zeta^{*}$ are $\left\{\left\{\varphi_{t}^{*}, \varsigma_{t}^{*}\right\}_{t=0}^{1}, \omega_{1}^{*}\right\}$. We set the candidate equilibrium price in the market economy to be $q$, as in $\xi^{C P}$, and set $f=B_{0}$. We then structure the debt limits and tax system so that in period 0 an agent with wealth $B_{0}\left(w_{0}\right)$ can afford to purchase each of the triples $\left\{C\left(\varphi_{0}^{*}\left(w_{0}, \theta\right)\right), Y\left(\varsigma_{0}^{*}\left(w_{0}, \theta\right)\right), B_{1}\left(\omega_{1}^{*}\left(w_{0}, \theta\right)\right)\right\}, \theta \in \Theta$, while, in period 1 , an agent with wealth $B_{1}\left(w_{1}\right)$ can afford each pair $\left\{C\left(\varphi_{1}^{*}\left(w_{1}, \theta\right)\right), Y\left(\varsigma_{1}^{*}\left(w_{1}, \theta\right)\right)\right\}, \theta \in \Theta$.

Under this arrangement, an agent with utility promise $w_{0}$ in the component planner economy is endowed with an initial quantity of claims equal to $B_{0}\left(w_{0}\right)$, the cost to a component planner of delivering $w_{0}$. The agent can then afford the constrained efficient allocation if she saves an amount equal to the component planner's continuation cost. This identification of an agent's savings with a component planner's costs is natural since the latter give the expected discounted net transfers to an agent under the constrained efficient allocation. It implies that an agent's wealth will convey information about the agent's past to the government. Specifically, if for all $w_{0}$ and $\theta$, the agent saves $B_{1}\left(\omega_{1}^{*}\left(w_{0}, \theta\right)\right)$, then the government will be able to infer the agent's continuation utility promise from $B_{1}$ and, hence, the continuation allocation to which the agent is entitled. Since the tax function will be designed to induce agents to choose this allocation, it will be essential that taxes depend on wealth and this informational role of wealth will crucially influence how wealth is taxed.

To simplify the notation we use the constrained efficient resource allocation rule, $\alpha^{*}=\left\{\left\{y_{t}^{*}, c_{t}^{*}\right\}_{t=0}^{1}, B_{1}^{*}\right\}$ associated with $\zeta^{*}$ to describe how the optimal tax system is constructed. In period 1, we set the tax 
functions so that for each $b \in B_{1}\left(\mathcal{W}_{1}\right)$ and $y \in \mathcal{Y}_{1}^{*}(b)$

$$
T_{1}(b, y)=b+y-c_{1}^{*}\left(b, \theta_{1}^{*}(b, y)\right),
$$

where $\theta_{1}^{*}(b, y) \in\left\{\theta: y_{1}^{*}(b, \theta)=y\right\}$. It then follows that an agent with savings $b \in B_{1}\left(\mathcal{W}_{1}\right)$ in the market economy can afford each of the period 1 allocations potentially available to an agent with period 1 component planner cost $b$ under $\alpha^{*}$. However, (21) only defines taxes for $\{b, y\} \in \operatorname{Graph} \mathcal{Y}_{1}^{*}$. To prevent agents from choosing savings in period 0 outside of $B_{1}\left(\mathcal{W}_{1}\right)=\left[B_{1}(\underline{U}), \infty\right)$, we simply impose the borrowing limit $\underline{b}=B_{1}(\underline{U})$ and set $\mathcal{B}_{1}=B_{1}\left(\mathcal{W}_{1}\right)$. For $b \in \mathcal{B}_{1}$ and $y \in \mathcal{Y} / \mathcal{Y}_{1}^{*}(b)$, we select taxes so that for each $\theta$ :

$$
u\left(c_{1}^{*}(b, \theta)\right)+\theta v\left(y_{1}^{*}(b, \theta)\right) \geq u\left(b+y-T_{1}(b, y)\right)+\theta v(y) .
$$

This ensures that no agent would choose such a labour supply. The constrained efficient utility allocation rule does not prescribe how this should be done, and we have some flexibility in selecting $T_{1}(b, \cdot)$ over these labour supplies. The procedure in period 0 is analogous, we set the tax function on Graph $\mathcal{Y}_{0}^{*}$ so that the agent can afford the relevant constrained efficient allocations. Specifically, for each $b \in \mathcal{B}_{0}=B_{1}\left(\mathcal{W}_{1}\right)$, we set

$$
T_{0}\left(b, y_{0}^{*}(b, \theta)\right)=b+y_{0}^{*}(b, \theta)-c_{0}^{*}(b, \theta)-q B_{1}^{*}(b, \theta) .
$$

As in $(22)$, we set taxes so that $y \in \mathcal{Y} / \mathcal{Y}_{0}^{*}(b)$ will not be chosen by an agent with initial wealth $b$.

Do these tax functions and borrowing limits succeed in implementing the constrained efficient allocation? To understand why they might fail, it is useful to compare the set of allocations available to an agent from different reporting strategies under $\alpha^{*}\left(\right.$ or $\left.\zeta^{*}\right)$ to those available to an agent in the market economy. Let $\mathcal{Z}^{C P}\left(b_{0}\right)$ denote the set of resource allocations available to an agent with initial planner cost $b_{0}$ under $\alpha^{*}$, or, equivalently, initial utility promise $B_{0}^{-1}\left(b_{0}\right)$ under $\zeta^{*}$. Let $\mathcal{Z}^{M E}\left(b_{0}\right)$ be the set of allocations available to an agent with initial wealth $b_{0}$ in the market economy. If $\mathcal{Z}^{M E}\left(b_{0}\right)=\mathcal{Z}^{C P}\left(b_{0}\right)$, then we can rely on the incentive compatibility of the constrained efficient utility allocation to ensure that this allocation is implemented. Since the constrained efficient allocation is affordable in the market economy under the candidate tax system, it follows that $\mathcal{Z}^{C P}\left(b_{0}\right) \subseteq \mathcal{Z}^{M E}\left(b_{0}\right)$. Deviations to choices outside of Graph $\mathcal{Y}_{t}^{*}$, $t=0,1$ are either ruled out directly by the borrowing limit or rendered undesirable by the tax system, as discussed above. Hence, we can restrict attention in the market economy to those allocations that remain within the graphs of $\mathcal{Y}_{t}^{*}, t=0,1$. These allocations comprise the set $\widetilde{\mathcal{Z}}^{M E}\left(b_{0}\right)$, where:

$$
\begin{gathered}
\widetilde{\mathcal{Z}}^{M E}\left(b_{0}\right): \equiv\left\{a=\left\{c_{t}, y_{t}\right\}_{t=0}^{1}: a \in \mathcal{Z}^{M E}\left(b_{0}\right) \text { and for } t=0,1,\left\{b_{t}, y_{t}\right\} \in \operatorname{Graph} \mathcal{Y}_{t}^{*}\right. \\
\text { where } \left.b_{1}=b_{0}+y_{0}-T\left(b_{0}, y_{0}\right)-c_{0}\right\} .
\end{gathered}
$$


Even after restricting attention to $\widetilde{\mathcal{Z}}^{M E}\left(b_{0}\right)$, there remain allocations available to the agent in the market economy that are unavailable to her under $\alpha^{*}$, so that $\mathcal{Z}^{C P}\left(b_{0}\right) \subset \widetilde{\mathcal{Z}}^{M E}\left(b_{0}\right)$. One or more of these may be preferred to the constrained efficient allocation. These allocations involve an agent selecting a labour supply $y=y_{0}^{*}(b, \theta)$ that is constrained efficient given the history $(b, \theta)$ and a savings level $B_{1}^{*}\left(b^{\prime}, y_{0}^{*}\left(b^{\prime}, \theta^{\prime}\right)\right)$ that is constrained efficient given some alternative history $\left(b^{\prime}, \theta^{\prime}\right)$. Since taxes are conditioned on current wealth and labour earnings only, the tax system cannot, in period 1, "look back" to the previous period's labour earnings and verify consistency with this period's wealth. Nor in period 0, can it prevent an agent from choosing a savings level that is inconsistent, from the point of view of $\alpha^{*}$, with that period's labour supply. Despite this, under the assumptions that an agent's preferences are separable in consumption and labour and shocks are i.i.d., our simple tax system works. The allocations in $\widetilde{\mathcal{Z}}^{M E}\left(b_{0}\right) / \mathcal{Z}^{C P}\left(b_{0}\right)$ are all inferior to the constrained efficient one.

Proposition 1 formally establishes the existence of a fiscal implementation for constrained efficient allocations. The main step of the proof involves splitting the period 0 problem of an agent in the market economy, and of a planner in the component planner economy, into two stages. In the market economy, an agent in the first stage of period 0 selects a labour supply $y_{0}$ and an after-tax quantity of resources, $x$. In the second stage, she allocates these resources between current consumption and savings. In the component planner economy, the planner first assigns utilities from labour supply, $\varsigma_{0}$, and interim utility promises, $d$. In the second stage of period 0 , she allocates $d$ between utility from consumption, $\varphi_{0}$ and a continuation utility promise, $\omega$. Figures A1 and A2 in the appendix display timelines for both economies.

Proposition 1 Let $\xi^{C P}=\left\{q, \zeta^{*},\left\{B_{t}\right\}_{t=0}^{1}, \Psi_{1}\right\}$ be an equilibrium of the component planner economy $\mathcal{E}^{C P}\left(\underline{U},\left\{G_{t}\right\}_{t=0}^{1}, \Psi_{0}\right)$. Then, the associated constrained efficient utility allocation can be implemented by a competitive equilibrium in a market economy with taxes and borrowing limits.

Proof: We directly construct a two period market economy with taxes and borrowing limits. We assume a market for claims opens in period 0 . We set $f=B_{0}$ and set $\Lambda_{0}$ to satisfy condition (1) in Definition 3. We set the government spending shocks to $\left\{G_{t}\right\}_{t=0}^{1}$, the debt limit to $\underline{b}=B_{0}(\underline{U})$ and $\mathcal{B}_{1}$ to $[\underline{b}, \infty)$. The (candidate) equilibrium price in the market economy is set to $q$. The proof will be complete if we can find taxes that ensure that for each $w_{0}$, an agent with initial wealth $B_{0}\left(w_{0}\right)$ in the market economy chooses the allocation $z\left(\zeta^{*}, w_{0}\right)$, where $\zeta^{*}=\left\{\left\{\varphi_{t}^{*}, \varsigma_{t}^{*}\right\}_{t=0}^{1}, \omega^{*}\right\}$.

The argument is in three steps that work back from period 1 to period 0 . In the first step, a tax function is found such that an agent with a stock of claims $B_{1}\left(w_{1}\right)$ in period 1 will choose the same allocation as 
is awarded to an agent with a utility promise of $w_{1}$ in the component planner economy. Period 0 is divided into two stages in both the market and the component planner economy. In the second stage, the intertemporal allocation of a given quantity of resources between time 0 consumption and claims is obtained. In the first stage, the labour-resource allocation is determined. The next step of the proof shows that the agent's second stage problem in the market economy is the dual of the corresponding second stage component planner's problem. In the final step, a tax function is found such that an agent with an initial stock of claims $B_{0}\left(w_{0}\right)$ chooses the same labour and resource pair as would be awarded to an agent with utility promise $w_{0}$ in the component planner economy. We give the argument for $\Theta=[\underline{\theta}, \bar{\theta}]$ and for $\varsigma_{t}^{*}\left(w_{0}, \cdot\right)$ continuous each $w_{0} \in \mathcal{W}_{0}$. These assumptions simplify the exposition; neither is essential.

PERIOD 1: A component planner with assigned utility promise $w_{1} \in \mathcal{W}_{1}$ solves the problem:

$$
B_{1}\left(w_{1}\right)=\inf _{\varphi: \Theta \rightarrow \mathcal{U}, \varsigma: \Theta \rightarrow \mathcal{V}} \int[C(\varphi(\theta))-Y(\varsigma(\theta))] d \pi
$$

s.t.

$$
\begin{gathered}
w_{1}=\int[\varphi(\theta)+\theta \varsigma(\theta)] d \pi \\
\forall \theta, \widetilde{\theta} \in \Theta, \varphi(\theta)+\theta \varsigma(\theta) \geq \varphi(\widetilde{\theta})+\theta \varsigma(\widetilde{\theta}) .
\end{gathered}
$$

Denote the policy functions that attain the infima in the problems $(24)$ by $\varsigma_{1}^{*}: \mathcal{W}_{1} \times \Theta \rightarrow \mathcal{V}$ and $\varphi_{1}^{*}$ : $\mathcal{W}_{1} \times \Theta \rightarrow \mathcal{U}$. Let $y_{1}^{*}$ and $c_{1}^{*}$ denote the corresponding constrained efficient resource allocation defined, as in Lemma 3 , by $y_{1}^{*}\left(B_{1}\left(w_{1}\right), \theta\right)=Y\left(\varsigma_{1}^{*}\left(w_{1}, \theta\right)\right)$ and $c_{1}^{*}\left(B_{1}\left(w_{1}\right), \theta\right)=C\left(\varphi_{1}^{*}\left(w_{1}, \theta\right)\right)$.

Next consider the first period problem of an agent in the market economy confronting a tax function $T_{1}$. An agent with wealth $b_{1} \in \mathcal{B}_{1}$ solves:

$$
V_{1}\left(b_{1}\right)=\sup _{c: \Theta \rightarrow \mathbb{R}_{+}, y: \Theta \rightarrow \mathcal{Y}} \int[u(c(\theta))+\theta v(y(\theta))] d \pi
$$

subject to the budget constraint, for each $\theta \in \Theta$,

$$
b_{1}=c(\theta)-y(\theta)+T_{1}\left(b_{1}, y(\theta)\right)
$$

Define $\mathcal{Y}_{1}^{*}(b)$ as in $(18)$ and for $b \in \mathcal{B}_{1}$ and $y \in \mathcal{Y}_{1}^{*}(b)$ set $T_{1}\left(b_{1}, y\right)$ according to $(21)$. Since $\Theta=[\underline{\theta}, \bar{\theta}]$ and $\varsigma_{1}^{*}\left(B_{1}^{-1}(b), \cdot\right)$ is continuous, $\mathcal{Y}_{1}^{*}(b)$ is an interval of the form $\left[\underline{y}_{1}(b), \bar{y}_{1}(b)\right]$ with $\underline{y}_{1}(b)=y_{1}^{*}(b, \bar{\theta})$ and $\bar{y}_{1}(b)=y_{1}^{*}(b, \underline{\theta})$. For $y>\bar{y}_{1}\left(b_{1}\right)$, set $T_{1}\left(b_{1}, y\right)>T_{1}\left(b_{1}, \bar{y}_{1}\left(b_{1}\right)\right)$ and such that $u\left(b_{1}+y-T_{1}\left(b_{1}, y\right)\right)+\underline{\theta} v(y)<$ $u\left(b_{1}+\bar{y}_{1}\left(b_{1}\right)-T_{1}\left(b_{1}, \bar{y}_{1}\left(b_{1}\right)\right)\right)+\underline{\theta} v\left(\bar{y}_{1}\left(b_{1}\right)\right)$. For example, extend $T_{1}\left(b_{1}, \cdot\right)$ linearly on $\mathcal{Y} / \mathcal{Y}_{1}^{*}\left(b_{1}\right)$ by setting 
$T_{1}\left(b_{1}, y\right)=T_{1}\left(b_{1}, \bar{y}_{1}\left(b_{1}\right)\right)+\bar{\tau}_{1}\left(y-\bar{y}_{1}\left(b_{1}\right)\right)$, where $\bar{\tau}_{1}=\left[u^{\prime}\left(c_{1}^{*}\left(b_{1}, \bar{y}_{1}\left(b_{1}\right)\right)\right)-\underline{\theta} v^{\prime}\left(\bar{y}_{1}\left(b_{1}\right)\right)\right] / u^{\prime}\left(c_{1}^{*}\left(b_{1}, \bar{y}_{1}\left(b_{1}\right)\right)\right)$. Similarly, for $y<\underline{y}_{1}\left(b_{1}\right)$, set $T_{1}\left(b_{1}, y\right)<T_{1}\left(b_{1}, \underline{y}_{1}\left(b_{1}\right)\right)$ and such that $u\left(b_{1}+y-T_{1}\left(b_{1}, y\right)\right)+\bar{\theta} v(y)<$ $u\left(b_{1}+\underline{y}_{1}\left(b_{1}\right)-T_{1}\left(b_{1}, \underline{y}_{1}\left(b_{1}\right)\right)\right)+\bar{\theta} v\left(\underline{y}_{1}\left(b_{1}\right)\right)$. For example, set $T_{1}\left(b_{1}, y\right)=T_{1}\left(b_{1}, \underline{y}_{1}\left(b_{1}\right)\right)+\underline{\tau}_{1}\left(y-\underline{y}_{1}\left(b_{1}\right)\right)$, where $\underline{\tau}_{1}=\left[u^{\prime}\left(c_{1}^{*}\left(b_{1}, \underline{y}_{1}\left(b_{1}\right)\right)\right)-\bar{\theta} v^{\prime}\left(\underline{y}_{1}\left(b_{1}\right)\right)\right] / u^{\prime}\left(c_{1}^{*}\left(b_{1}, \underline{y}_{1}\left(b_{1}\right)\right)\right)$.

Consider an agent in the market economy in period 1 with wealth $b_{1}=B_{1}\left(w_{1}\right)$ and shock $\theta$. Under (21), if the agent chooses labour $y_{1}^{*}\left(b_{1}, \theta^{\prime}\right) \in \mathcal{Y}_{1}^{*}\left(b_{1}\right)$, she obtains consumption $c_{1}^{*}\left(b_{1}, \theta^{\prime}\right)$. By construction, this provides the utility pair $\left(\varphi_{1}^{*}\left(w_{1}, \theta^{\prime}\right), \varsigma_{1}^{*}\left(w_{1}, \theta^{\prime}\right)\right)$. By choosing different labour levels in $\mathcal{Y}_{1}^{*}\left(b_{1}\right)$, the agent can obtain the entire set of period 1 report-contingent resource allocations available to an agent with utility promise $w_{1}$ in the component planner economy. Incentive compatibility implies that amongst these, the agent obtains the highest payoff from $\left(c_{1}^{*}\left(b_{1}, \theta\right), y_{1}^{*}\left(b_{1}, \theta\right)\right)$. For $y \in \mathcal{Y} / \mathcal{Y}_{1}^{*}\left(b_{1}\right)$, consider first an agent choosing $y=\bar{y}_{1}\left(b_{1}\right)+\delta, \delta>0$. By construction, $u\left(b_{1}+y-T_{1}\left(b_{1}, y\right)\right)+\theta v(y)<u\left(b_{1}+\bar{y}_{1}\left(b_{1}\right)-\right.$ $\left.T_{1}\left(b_{1}, \bar{y}_{1}\left(b_{1}\right)\right)\right)+\theta v\left(\bar{y}_{1}\left(b_{1}\right)\right)+(\theta-\underline{\theta})\left(v(y)-v\left(\bar{y}_{1}\left(b_{1}\right)\right)\right)<u\left(b_{1}+\bar{y}_{1}\left(b_{1}\right)-T_{1}\left(b_{1}, \bar{y}_{1}\left(b_{1}\right)\right)\right)+\theta v\left(\bar{y}_{1}\left(b_{1}\right)\right)$. Thus, for all $y>\bar{y}_{1}\left(b_{1}\right)$, the agent is better off reducing her labour to $\bar{y}_{1}\left(b_{1}\right)$. By a similar argument the agent would never choose $y<\underline{y}_{1}\left(b_{1}\right)$. It follows that the agent will choose the allocation $\left(c_{1}^{*}\left(b_{1}, \theta\right), y_{1}^{*}\left(b_{1}, \theta\right)\right)$ and, hence, the utility pair $\left(\varphi_{1}^{*}\left(w_{1}, \theta\right), \varsigma_{1}^{*}\left(w_{1}, \theta\right)\right)$. Since $b_{1}$ and $\theta$ were arbitrary, it follows that for all $b_{1}^{\prime} \in \mathcal{B}_{1}$ and $\theta^{\prime} \in \Theta$, an agent will choose $\left(c_{1}^{*}\left(b_{1}^{\prime}, \theta^{\prime}\right), y_{1}^{*}\left(b_{1}^{\prime}, \theta^{\prime}\right)\right)$ when confronted with the tax function $T_{1}$. The agent's value function in the market economy, $V_{1}$, defined in (25), then equals $B_{1}^{-1}$ and, hence, is strictly increasing.

PERIOD 0: We divide the agent's problem in the market economy into two stages. In the first, the agent chooses labour $y_{0}$ and resources $x$. In the second, she allocates $x$ between current consumption $c_{0}$ and claims $b_{1}$. Similarly, the component planner's problem can be subdivided. In the first stage, the agent reports her shock and receives a utility from labor, $\varsigma_{0}$, and an interim utility promise $d$. In the second stage, the planner allocates the interim promise between utility from current consumption $\varphi_{0}$ and a continuation utility promise $w_{1}$.

PERIOD 0, SECOND STAGE: Consider the second stage problem of a component planner with interim utility promise $d \in \mathcal{D}=\left\{\varphi+\beta w: \varphi \in \mathcal{U}, w \in \mathcal{W}_{1}\right\}$ :

$$
X(d)=\inf _{\varphi_{0} \in \mathcal{U}, w_{1} \in \mathcal{W}_{1}} C\left(\varphi_{0}\right)+q B_{1}\left(w_{1}\right)
$$

subject to: $d=\varphi_{0}+\beta w_{1}$. The constraint set for each problem (26) is compact and the objective is continuous. Hence, it has a solution. Also, $X$ is strictly increasing. Let $\widetilde{\varphi}_{0}^{*}: \mathcal{D} \rightarrow \mathcal{U}$ and $\widetilde{\omega}^{*}: \mathcal{D} \rightarrow \mathcal{W}_{1}$ denote policy functions that attain the infima in the problems (26). Define the corresponding resource 
allocation functions by $\widetilde{c}_{0}^{*}$ and $\widetilde{b}_{1}^{*}$, where $\widetilde{c}_{0}^{*}(X(d))=C\left(\widetilde{\varphi}_{0}^{*}(d)\right)$ and $\widetilde{b}_{1}^{*}(X(d))=B_{1}\left(\widetilde{\omega}^{*}(d)\right)$.

Next consider the agent's second stage problem in the market economy. The agent allocates $x \geq q \underline{b}$ units of resources across current consumption and savings to solve:

$$
D(x)=\sup _{c_{0} \in \mathbb{R}_{+}, b_{1} \geq \underline{b}} u\left(c_{0}\right)+\beta V_{1}\left(b_{1}\right)
$$

subject to $x=c_{0}+q b_{1}$. The allocation $\left(\widetilde{c}_{0}^{*}(x), \widetilde{b}_{1}^{*}(x)\right)$ is optimal for the agent in this problem. To see this suppose that there was some alternative allocation $\left(c^{\prime}, b^{\prime}\right)$ such that $x=c^{\prime}+q b^{\prime}, c^{\prime} \in \mathbb{R}_{+}, b^{\prime} \geq \underline{b}$ and $u\left(c^{\prime}\right)+\beta V_{1}\left(b^{\prime}\right)>d=X^{-1}(x)$. Then, since $d \in \mathcal{D}$, and $u$ and $V_{1}$ are continuous and monotone, there exists an allocation $\left(c^{+}, b^{+}\right)$with $c^{+} \leq c^{\prime}$ and $b^{+} \leq b^{\prime}$ with at least one of these inequalities strict such that $u\left(c^{+}\right)+\beta V_{1}\left(b^{+}\right)=d$. But then $\left(u\left(c^{+}\right), V_{1}\left(b^{+}\right)\right)$attains the interim utility promise $d$ and has a cost strictly less than $x$. This contradicts the optimality of $\widetilde{\varphi}_{0}^{*}$ and $\widetilde{\omega}^{*}$ at $d$ for the component planner's problem.

PERIOD 0, FIRST STAGE: In this stage, a component planner utility promise $w_{0} \in \mathcal{W}_{0}$ solves:

$$
B_{0}\left(w_{0}\right)=\inf _{d: \Theta \rightarrow \mathcal{D}, \varsigma: \Theta \rightarrow \mathcal{V}} \int[X(d(\theta))-Y(\varsigma(\theta))] d \pi
$$

s.t.

$$
\begin{gathered}
w_{0}=\int[X(d(\theta))+\theta \varsigma(\theta)] d \pi, \\
\forall \theta, \theta^{\prime} \in \Theta, d(\theta)+\theta \varsigma(\theta) \geq d\left(\theta^{\prime}\right)+\theta \varsigma\left(\theta^{\prime}\right) .
\end{gathered}
$$

Denote the policy functions that attain the infima in these problems by $d_{0}^{*}: \mathcal{W}_{0} \times \Theta \rightarrow \mathcal{D}$ and $\varsigma_{0}^{*}:$ $\mathcal{W}_{0} \times \Theta \rightarrow \mathcal{V}$. Let $y_{0}^{*}\left(B_{0}\left(w_{0}\right), \theta\right)=Y\left(\varsigma_{0}^{*}\left(w_{0}, \theta\right)\right)$ and $x_{0}^{*}\left(B_{0}\left(w_{0}\right), \theta\right)=X\left(d_{0}^{*}\left(w_{0}, \theta\right)\right)$.

Next consider the first stage problem of an agent in the market economy with initial wealth $b_{0} \in \mathcal{B}_{0}$, $\mathcal{B}_{0}=B_{0}\left(\mathcal{W}_{0}\right)$ under a tax function $T_{0}$ :

$$
V_{0}\left(b_{0}\right)=\sup _{x c: \Theta \rightarrow \mathbb{R}_{+}, y: \Theta \rightarrow \mathcal{Y}} \int[D(x(\theta))+\theta v(y(\theta))] d \pi
$$

subject to the budget constraint, for each $\theta \in \Theta$,

$$
b_{0}=x(\theta)-y(\theta)+T_{0}(b, y(\theta)) .
$$

Define $\mathcal{Y}_{0}^{*}(b)$ as in (18). For each $b_{0} \in \mathcal{B}_{0}$, and $\theta$, set

$$
T_{0}\left(b_{0}, y\left(b_{0}, \theta\right)\right)=b_{0}+y\left(b_{0}, \theta\right)-x_{0}^{*}\left(b_{0}, \theta\right) \text {. }
$$


Since $\Theta=[\underline{\theta}, \bar{\theta}]$ and $\varsigma_{0}^{*}\left(B_{0}^{-1}(b), \cdot\right)$ is continuous, $\mathcal{Y}_{0}^{*}(b)$ is an interval of the form $\left[\underline{y}_{0}(b), \bar{y}_{0}(b)\right]$. For $y>\bar{y}_{0}\left(b_{0}\right)$, set $T_{0}\left(b_{0}, y\right)>T_{0}\left(b_{0}, \bar{y}_{0}\left(b_{0}\right)\right)$ and such that $D\left(b_{0}+y-T_{0}\left(b_{0}, y\right)\right)+\underline{\theta} v(y)<D\left(b_{0}+\bar{y}_{0}\left(b_{0}\right)-T_{0}\left(b_{0}, \bar{y}_{0}\left(b_{0}\right)\right)\right)$ $+\underline{\theta} v\left(\bar{y}_{0}\left(b_{0}\right)\right)$. Similarly, for $y<\underline{y}_{0}\left(b_{0}\right)$, set $T_{0}\left(b_{0}, y\right)<T_{0}\left(b_{0}, \underline{y}_{0}\left(b_{0}\right)\right)$ and such that $D\left(b_{0}+y-T_{0}\left(b_{0}, y\right)\right)$ $+\bar{\theta} v(y)<D\left(b_{0}+\underline{y}_{0}\left(b_{0}\right)-T_{0}\left(b_{0}, \underline{y}_{0}\left(b_{0}\right)\right)\right)+\bar{\theta} v\left(\underline{y}_{0}\left(b_{0}\right)\right)$. Then, the set of budget feasible labour and resource combinations, $\left(x_{0}, y_{0}\right)$, for an agent with initial wealth $b_{0}=B_{0}\left(w_{0}\right)$ and shock $\theta_{0}$ in the market economy includes those available to an agent with initial promise $w_{0}$ in the component planner problem. By incentive-compatibility, $\left(x_{0}^{*}\left(b_{0}, \theta\right), y_{0}^{*}\left(b_{0}, \theta\right)\right)$ is optimal for the agent amongst these. In the market economy, the agent can also increase her labour above $\bar{y}_{0}\left(b_{0}\right)$ or reduce it below $\underline{y}_{0}\left(b_{0}\right)$. However, as in period 1 , allocations obtained in this way are sub-optimal. Let $c_{0}^{*}\left(b_{0}, \theta\right)=\widetilde{c}_{0}^{*}\left(x_{0}^{*}\left(b_{0}, \theta\right)\right)$ and $b^{*}\left(b_{0}, \theta\right)=$ $\widetilde{b}_{1}^{*}\left(x_{0}^{*}\left(b_{0}, \theta\right)\right)$.

Combining the previous arguments, an agent who confronts the price $q$ and the tax system $\left\{T_{0}, T_{1}\right\}$, who is endowed with a stock of claims $b_{0}=B_{0}\left(w_{0}\right)$ and who receives the shock $\theta$ will select an initial labor and resource allocation equal to the allocation $\left(y_{0}^{*}\left(b_{0}, \theta\right), x_{0}^{*}\left(b_{0}, \theta\right)\right)$. Next, the agent allocates resources in each state $\theta, x_{0}^{*}\left(b_{0}, \theta\right)$, intertemporally between current consumption and claims. Her choices, will coincide with those made by the component planner: $c_{0}^{*}\left(b_{0}, \theta\right)$ and $b^{*}\left(b_{0}, \theta\right)$. Finally, the agent enters period 1 , with wealth $b^{*}\left(b_{0}, \theta\right)$, receives the period 1 shock and makes consumption and labour choices that once again match those of the component planner. Hence, an agent's optimal policy functions in the market economy evaluated at an initial wealth $b_{0}=B_{0}\left(w_{0}\right)$ induce the allocation $z\left(\zeta^{*}, w_{0}\right)$.

Remark 1: The key step in this proof is the decomposition of the agent's and the component planner's period 0 problem into two stages, where the only link between these stages is the quantity of resources, $x_{0}^{*}$, or interim utility promise, $d_{0}^{*}$, that is passed from the first to the second. Step 2 of the proof shows that although the intertemporal allocation of resources is made by the agent in the market economy, when given the appropriate quantity of resources, she makes the same choice as the component planner.

The simple decomposition that underlies this argument is disrupted if, given the quantity of resources $x_{0}^{*}$ or the interim utility promise $d_{0}^{*}$, the agent's intertemporal marginal rate of substitution in the second stage of period 0 depends upon either her labour supply or her preference shock in the first stage. The first case occurs when preferences are non-separable in labour and consumption, the second when the preference shock $\theta$ is persistent. In both cases, neither $x_{0}^{*}$ nor $d_{0}^{*}$ is a sufficient state variable for the stage 2 intertemporal allocation problem and the decomposition fails. We return to the persistent shock case in the concluding remarks. 
Remark 2: Our definition of a fiscal implementation requires only that, when given an appropriate initial endowment of wealth, agents choose the allocations induced by the constrained-efficient utility allocation rule $\zeta^{*}$. The tax functions constructed above do more than this. They implement the complete rule $\zeta^{*}$. To see the difference, let $\mathcal{B}_{1}^{*}: \equiv\left\{b_{1} \in \mathcal{B}_{1}: b_{1}=B_{1}^{*}\left(b_{0}, y_{0}\right),\left(b_{0}, y_{0}\right) \in\right.$ Graph $\left.\mathcal{Y}_{0}^{*}\right\}$ denote the set of possible period 1 continuation costs for the component planner under $\zeta^{*}$. Recall that Graph $\mathcal{Y}_{0}^{*}$ equals all those initial planner cost and labour supply pairs that occur under $\zeta^{*}$. The initial distribution of wealths and the period 0 tax function in our fiscal implementation imply that each agent should select a savings level $b_{1} \in \mathcal{B}_{1}^{*}$. The tax system constructed in the above proof induces an agent with savings $b_{1}$ to choose the period 1 allocation $\left\{c_{1}^{*}\left(b_{1}, \cdot\right), y_{1}^{*}\left(b_{1}, \cdot\right)\right\}$ prescribed by the utility allocation rule $\zeta^{*}$. It does this at every $b_{1} \in \mathcal{B}_{1}$ and not just at those in $\mathcal{B}_{1}^{*}$. This is sufficient for implementation and conveniently pins down $T_{1}$ for all savings levels. It is also informationally parsimonious since the government does not need to know $\mathcal{B}_{1}^{*}$ in period 1.

However, if the government did know $\mathcal{B}_{1}^{*}$, it could use this information to design an even simpler tax system. Specifically, if an agent entered period 1 with wealth $b_{1} \notin \mathcal{B}_{1}^{*}$, it would be immediately revealed to the government that the agent had taken actions inconsistent with the constrained efficient allocation. The agent could then be penalised in the same way that agents who choose savings levels and labour supplies outside of Graph $\mathcal{Y}_{t}^{*}$ are penalised by the tax system constructed in the proof. In short, it is not important what allocation an agent receives if she chooses $b_{1} \notin \mathcal{B}_{1}^{*}$, provided that that allocation discourages her from choosing such a $b_{1}$ in the first place. We construct examples along these lines in Section 5.

Remark 3: An immediate implication of $(27)$ and (29) is that, for each $b_{0}=B_{0}\left(w_{0}\right)$ :

$$
E\left[T_{0}\left(b_{0}, y_{0}^{*}\left(b_{0}, \theta_{0}\right)\right) \mid b_{0}\right]=E\left[b_{0}+y_{0}^{*}\left(b_{0}, \theta_{0}\right)-x_{0}^{*}\left(b_{0}, y_{0}^{*}\left(b_{0}, \theta_{0}\right)\right) \mid b_{0}\right]=0
$$

Similarly at date $1, E\left[T_{1}\left(b_{1}, y_{1}^{*}\left(b_{1}, \theta_{1}\right)\right) \mid b_{1}\right]=0$. Thus, the tax system is solely redistributive and raises no revenue to finance the government spending levels $\left\{G_{t}\right\}_{t=0}^{1}$. In our implementation, such spending is financed via an appropriate setting of the initial distribution of claims. In particular, to extract net resources from the population of agents, the government must hold claims against them at date 0 .

\subsection{Implementation in economies of arbitrary finite length.}

The argument in Proposition 1 is easily extended to economies of arbitrary finite length. The proof involves a straightforward iteration on the argument in Proposition 1. 
Proposition 2 Let $\xi^{C P}$ be an equilibrium of the component planner economy $\mathcal{E}^{C P}\left(\left\{\underline{U}_{t+1}\right\}_{t=0}^{T-1},\left\{G_{t}\right\}_{t=0}^{T}, \Psi_{0}\right)$. Then, the associated constrained efficient utility allocation can be implemented by a competitive equilibrium in a market economy with taxes and borrowing limits.

\section{The Optimal Pattern of Wedges}

We now obtain the key properties of constrained-efficient allocations. In particular, we characterise their implications for the pattern of wedges between individual and social marginal rates of substitution and marginal rates of transformation. These properties are well known in the literature and we proceed heuristically. However, they are an essential precursor to the analysis of optimal tax functions that follows.

For expositional ease, we focus on the case $\Theta=[\underline{\theta}, \bar{\theta}]$ and assume that the functions that comprise the optimal utility allocation rule, $\zeta^{*}=\left\{\left\{\varphi_{t}^{*}, \varsigma_{t}^{*}, \omega_{t+1}^{*}\right\}_{t=0}^{T-1}, \varphi_{T}^{*}, \varsigma_{T}^{*}\right\}$, are piecewise differentiable in $\theta \cdot{ }^{12} \mathrm{We}$ assume that $\pi$ admits a continuous density $\rho$. As a notational convention, if $x: \Theta \rightarrow \mathbb{R}$ is a piecewise differentiable function, then we denote its derivative at $\theta$ by $\dot{x}(\theta)$. Define the function $U: \Theta \rightarrow \mathbb{R}$ by:

$$
U(\theta): \equiv \varphi(\theta)+\theta \varsigma(\theta)+\beta \omega(\theta)
$$

By standard arguments, for example Salanié (1997), $\{\varphi, \varsigma, \omega\}$ is incentive compatible if and only if:

$$
\begin{aligned}
\dot{U}(\theta)= & \varsigma(\theta) \quad \text { a.e. } \theta, \\
& \varsigma(\theta) \text { non-decreasing in } \theta .
\end{aligned}
$$

We formulate the period $t \in\{0, \ldots, T-1\}$ problem of a component planner with utility promise $w_{t}$ as an optimal control problem. ${ }^{13}$ To obtain the component planner's period $t$ Hamiltonian, we use (31) to replace $\varphi$ with $U$ and we drop the constraint that $\varsigma(\theta)$ is non-decreasing ${ }^{14}$ :

$$
\begin{aligned}
H_{t}^{w_{t}}(U, \varsigma, \omega ; \chi, \lambda, \phi)(\theta) & =-\chi\left(U(\theta)-w_{t}\right) \rho(\theta)-\lambda(\theta) \varsigma(\theta)+ \\
& {\left[C(U(\theta)-\theta \varsigma(\theta)-\beta \omega(\theta))-Y(\varsigma(\theta))+q_{t} B_{t+1}(\omega(\theta))\right] \rho(\theta)+\phi(\theta)\left(\omega(\theta)-\underline{U}_{t}\right) \rho(\theta) . }
\end{aligned}
$$

Here $\chi$ is the multiplier on the promise-keeping constraint $w_{t}=\int_{\underline{\theta}}^{\bar{\theta}} U(\theta) \rho(\theta) d \theta, \lambda$ is the costate variable associated with the incentive compatibility constraint $(32)$ and $\phi(\theta)$ is the multiplier on the constraint

\footnotetext{
${ }^{12}$ Kahn (1993) provides conditions for an optimal static mechanism to be absolutely continuous.

${ }^{13}$ The period $T$ problem can be similarly formulated. For brevity we omit it.

${ }^{14}$ See Salanié (1997) for sufficient conditions to ensure that it is not binding.
} 
$\omega(\theta) \geq \underline{U}_{t}$. The component planner's problem in period $t$ can then be written as:

$$
B_{t}\left(w_{t}\right)=\inf _{\{\chi, \lambda, \phi\}} \sup _{\{U, \varsigma, \omega\}} \int H_{t}^{w_{t}}(U, \varsigma, \omega ; \chi, \lambda, \phi)(\theta) d \theta .
$$

Denote the optimal multipliers by $\left\{\chi_{t}^{*}, \lambda_{t}^{*}, \phi_{t}^{*}\right\}$. The first order conditions from (34) imply a particular pattern of wedges. We describe this pattern in terms of the constrained efficient resource allocation rule.

ThE InSURANCE WEDGE: The first order condition for $U(\theta)$ at $t$, re-expressed in terms of consumption is:

$$
\dot{\lambda}_{t}^{*}\left(w_{t}, \theta\right)=-\left[\chi_{t}^{*}\left(w_{t}\right)-\frac{1}{u^{\prime}\left(c_{t}^{*}\left(b_{t}, \theta\right)\right)}\right] \rho(\theta),
$$

where $b_{t}=B_{t}\left(w_{t}\right)$. Thus, if $\dot{\lambda}_{t}^{*}\left(w_{t}, \cdot\right) / \rho(\cdot)$ is non-constant then an agent's marginal utility of consumption is not equated across states and insurance against preference shocks is imperfect. As observed in Lemma 3, $\varphi_{t}^{*}$, and, hence, $c_{t}^{*}$ are monotone in $\theta$. Strict monotonicity stems from the binding incentive-compatibility constraint. $^{15}$

THE EFFORT WEDGE: The first order conditions for $U(\theta)$ and $\varsigma(\theta)$ at $t$, re-expressed in terms of consumption and labour imply:

$$
\frac{-\theta v^{\prime}\left(y_{t}^{*}\left(b_{t}, \theta\right)\right)}{u^{\prime}\left(c_{t}^{*}\left(b_{t}, \theta\right)\right)}=1+\frac{\lambda_{t}^{*}\left(w_{t}, \theta\right) v^{\prime}\left(y_{t}^{*}\left(b_{t}, \theta\right)\right)}{\rho(\theta)}
$$

where $b_{t}=B_{t}\left(w_{t}\right)$. Since the marginal rate of transformation of labour into output is $1,-\frac{\lambda_{t}^{*}\left(w_{t}, \theta\right) v^{\prime}\left(y_{t}^{*}\left(b_{t}, \theta\right)\right)}{\rho(\theta)}$ represents the wedge between this marginal rate of transformation and the agent's marginal rate of substitution. When $\lambda_{t}^{*}\left(w_{t}, \theta\right)>0$, it is positive.

THE INTERTEMPoral Wedge: Combining the first order conditions for $U(\theta), \omega(\theta)$ and the envelope condition from the component planner's first period problem yields the following inverted Euler equation:

$$
\frac{1}{u^{\prime}\left(c_{t}^{*}\left(b_{t}, \theta\right)\right)}=\frac{q_{t}}{\beta} E_{\theta^{\prime}}\left[\frac{1}{u^{\prime}\left(c_{t+1}^{*}\left(B_{t+1}^{*}\left(b_{t}, \theta\right), \theta^{\prime}\right)\right)}\right]+\phi_{t}^{*}\left(w_{t}, \theta\right),
$$

where $b_{t}=B_{t}\left(w_{t}\right)$. Assuming that the lower bound on $\omega_{t+1}^{*}\left(w_{t}, \theta\right)$ does not bind and $\phi_{t}^{*}\left(w_{t}, \theta\right)=0$, we have, by Jensen's inequality,

$$
q_{t} \leq \beta E_{t}\left[\frac{u^{\prime}\left(c_{t+1}^{*}\left(B_{t+1}^{*}\left(b_{t}, \theta\right), \theta^{\prime}\right)\right)}{u^{\prime}\left(c_{t}^{*}\left(b_{t}, \theta\right)\right)}\right] .
$$

\footnotetext{
${ }^{15}$ The transversality condition on $U$ implies that $\lambda_{t}^{*}\left(w_{t}, \bar{\theta}\right)=\lambda_{t}^{*}\left(w_{t}, \underline{\theta}\right)=0$. From Lemma $3, c_{t}^{*}$ is non-increasing. It follows that $\dot{\lambda}_{t}^{*}\left(w_{t}\right)$ is non-increasing and if $\lambda_{t}^{*}\left(w_{0}, \theta\right) \neq 0$ for some $\theta$, then $\dot{\lambda}_{t}^{*}\left(w_{0}, \cdot\right)$ is non-constant.
} 
This inequality is strict if $u^{\prime}\left(c_{t+1}^{*}\left(B_{t+1}^{*}\left(b_{t}, \theta\right), \cdot\right)\right)$ is non-constant, which is the case in this problem when the incentive-compatibility constraint binds. Thus, there is a wedge between the social intertemporal marginal rate of substitution $q_{t}$ and the individual agent's intertemporal marginal rate of substitution $\beta E_{\theta^{\prime}}\left[u^{\prime}\left(c_{t+1}^{*}\left(B_{t+1}^{*}\left(b_{t}, \theta\right), \theta^{\prime}\right)\right)\right] / u^{\prime}\left(c_{t}^{*}\left(b_{t}, \theta\right)\right)$.

The insurance and effort wedges occur in each period, and the resulting implications for optimal nonlinear income taxation are well known from the static public finance literature based on Mirrlees (1971). If a consumption-labour allocation $\left(c_{t}^{*}\left(b_{t}, \theta\right), y_{t}^{*}\left(b_{t}, \theta\right)\right)$ is to be implemented at date $t$ with the tax function $T_{t}$, it must be such that:

$$
\begin{aligned}
\left(c_{t}^{*}\left(b_{t}, \theta\right), y_{t}^{*}\left(b_{t}, \theta\right)\right) & \in \underset{c, y}{\arg \sup } u(c)+\theta v(y) \\
\text { s.t. } c+T_{t}\left(b_{t}, y\right) & =y+b_{t}-q_{t+1} B_{t+1}^{*}\left(b_{t}, \theta\right) .
\end{aligned}
$$

In particular, if $T_{t}\left(b_{t}, \cdot\right)$ is differentiable at $y_{t}^{*}\left(b_{t}, \theta\right)$ :

$$
\frac{\partial T_{t}\left(b_{t}, y_{t}^{*}\left(b_{t}, \theta\right)\right)}{\partial y}=-\frac{\lambda_{t}^{*}\left(w_{t}, \theta\right) v^{\prime}\left(y_{t}^{*}\left(b_{t}, \theta\right)\right)}{\rho(\theta)}
$$

where $b_{t}=B_{t}\left(w_{t}\right)$ and the marginal income tax at $\left(b_{t}, y_{t}^{*}\left(b_{t}, \theta\right)\right)$ equals the effort wedge.

The intertemporal wedge was first derived by Diamond and Mirrlees (1978) and Rogerson (1985). Golosov, Kocherlakota and Tsyvinski (2003) establish that this wedge is present in a very large class of private information economies. This wedge stems from the adverse effect of savings on incentives. Higher saving at date $t$ exacerbates the incentive problem at $t+1$, since it reduces the correlation between an agent's consumption and her labour supply in that period. The intertemporal wedge adjusts for this additional marginal social cost of saving. Just as the effort wedge gives rise to positive marginal labour income taxes, it is natural to infer that the intertemporal wedge provides a rationale for asset taxation. Specifically, implementation of the socially optimal intertemporal consumption and savings allocation requires that:

$$
\begin{aligned}
& \left\{c_{t}^{*}\left(b_{t}, \theta\right), B_{t+1}^{*}\left(b_{t}, \theta\right), c_{t+1}^{*}\left(B_{t+1}^{*}\left(b_{t}, \theta\right), \cdot\right)\right\} \in \arg \max _{c_{t}, b_{t+1}, c_{t+1}} u\left(c_{t}\right)+\beta E_{t} u\left(c_{t+1}\right) \\
& \text { s.t. } \quad c_{t}+T_{t}\left(b_{t}, y_{t}^{*}\left(b_{t}, \theta\right)\right)=y_{t}^{*}\left(b_{t}, \theta\right)+b_{t}-q_{t} b_{t+1} \text {, }
\end{aligned}
$$

and $\forall \theta^{\prime}, \quad c_{t+1}\left(\theta^{\prime}\right)+T_{t+1}\left(b_{t+1}, y_{t+1}^{*}\left(B_{t+1}^{*}\left(b_{t}, \theta\right), \theta^{\prime}\right)\right)=y_{t+1}^{*}\left(B_{t+1}^{*}\left(b_{t}, \theta\right), \theta^{\prime}\right)+b_{t+1}-q_{t+1} B_{t+2}^{*}\left(B_{t+1}^{*}\left(b_{t}, \theta\right), \theta^{\prime}\right)$.

Hence, assuming that the function $T_{t+1}$ is differentiable in its first argument, $T_{t+1}$ must be consistent with the agent's Euler equation holding at the constrained efficient allocation:

$$
q_{t}=\beta E_{t}\left[\left(1-\frac{\partial T_{t+1}\left(B_{t+1}^{*}\left(b_{t}, \theta\right), y_{t+1}^{*}\left(B_{t+1}^{*}\left(b_{t}, \theta\right), \theta^{\prime}\right)\right)}{\partial b}\right) \frac{u^{\prime}\left(c_{t+1}^{*}\left(B_{t+1}^{*}\left(b_{t}, \theta\right)\right), \theta^{\prime}\right)}{u^{\prime}\left(c_{t}^{*}\left(b_{t}, \theta\right)\right)}\right] .
$$


A positive intertemporal wedge requires:

$$
0<E_{t}\left[\frac{\partial T_{t+1}}{\partial b} \frac{u^{\prime}\left(c_{t+1}^{*}\right)}{u^{\prime}\left(c_{t}^{*}\right)}\right]=E_{t}\left[\frac{\partial T_{t+1}}{\partial b}\right] E_{t}\left[\frac{u^{\prime}\left(c_{t+1}^{*}\right)}{u^{\prime}\left(c_{t}^{*}\right)}\right]+\operatorname{Cov}_{t}\left[\frac{\partial T_{t+1}}{\partial b}, \frac{u^{\prime}\left(c_{t+1}^{*}\right)}{u^{\prime}\left(c_{t}^{*}\right)}\right] .
$$

This decomposition of the effect of marginal taxation of wealth on the intertemporal trade-off illustrates two ways in which asset taxation can generate an intertemporal wedge. The most direct way is to reduce the expected return on savings with a positive expected marginal asset tax $E_{t}\left[\frac{\partial T_{t+1}}{\partial b}\right]>0$. The second way is to set marginal taxes on wealth so that the after-tax return on savings covaries positively with consumption, generating $\operatorname{Cov}_{t}\left[\frac{\partial T_{t+1}}{\partial b}, \frac{u^{\prime}\left(c_{t+1}^{*}\right)}{u^{\prime}\left(c_{t}^{*}\right)}\right]>0$. This discourages savings in period $t$ by making noncontingent claims a less effective hedge against period $t+1$ consumption risk. It also boosts incentives in period $t+1$ by reinforcing the covariance between consumption and labour income. Crucially, (40) shows that there is no presumption that the intertemporal wedge translates into positive expected marginal asset taxes. In addition, for the after-tax return on assets to covary with labour earnings and consumption, the marginal asset tax must be stochastic. Specifically, it must depend on labour earnings in period $t+1$, which are uncertain when the savings choice is made in period $t$.

We explore these issues in the following section. We present several examples that reveal the respective roles of the expected and the stochastic components of marginal asset taxation in generating the intertemporal wedge. The first example shows that it is essential that marginal asset taxes generate a positive covariance between after-tax returns on assets and labour earnings. Absent this covariance, an agent could increase her lifetime utility with a deviation in which she saves more in period $t$ and reduces her labour supply in all states in period $t+1$, relative to the constrained efficient allocation. Moreover, in this first example, the optimal tax system implies that the expected marginal asset tax is equal to zero. The subsequent examples illustrate the link between the dependence of the constrained-efficient labour allocation in period $t+1$ on wealth and a positive expected marginal asset tax. Lastly, we discuss the role of the lower bound on continuation utility.

\section{$5 \quad$ Revealing Examples}

We now present three examples in order of complexity of the physical environment. As we make the physical environment more complicated, the information conveyed by an agent's wealth changes, leading to corresponding changes in the tax system. 


\subsection{Example 1: Zero Expected Marginal Asset Tax}

For our first two examples, we consider a stripped down version of the model in the previous section. To focus attention on the role of marginal asset taxation, we assume that in period 0 an agent consumes, but does not work. In period 1 , the agent receives a preference shock from $\Theta=\{\underline{\theta}, \bar{\theta}\}, \underline{\theta}<\bar{\theta}$, chooses a labour supply from the discrete set $\mathcal{Y}=\{\underline{y}, \bar{y}\}, \underline{y}<\bar{y}$ and consumes.

Component planner's problem. The representative component planner faces an equilibrium intertemporal price $q$ and solves the following problem ${ }^{16}$ :

$$
\begin{array}{lll}
\text { Period } 0 & B_{0}\left(w_{0}\right)= & \inf _{\{\varphi, \omega\}} C(\varphi)+q B_{1}(\omega) \\
\text { s.t. } & w_{0}=\varphi+\beta \omega
\end{array}
$$

and

$$
\begin{array}{lll}
\text { Period } 1 \quad B_{1}\left(w_{1}\right)= & \inf _{\{\varphi, \varsigma\}} \sum_{\theta \in \Theta}\{C(\varphi(\theta))-Y(\varsigma(\theta))\} \pi(\theta) \\
\text { s.t. } & w_{1}=\sum_{\theta \in \Theta}\{\varphi(\theta)+\theta \varsigma(\theta)\} \pi(\theta) \\
& \forall \theta, \theta^{\prime}, \quad \varphi(\theta)+\theta \varsigma(\theta) \geq \varphi\left(\theta^{\prime}\right)+\theta \varsigma\left(\theta^{\prime}\right) .
\end{array}
$$

Following the notation in previous sections, let $\zeta^{*}=\left\{\varphi_{0}^{*}, \varphi_{1}^{*}, \varsigma_{1}^{*}, \omega^{*}\right\}$ denote the solution to these problems. A solution $\zeta^{*}$ and the value function $B_{1}$ have the following properties.

Lemma 4 Assume that $C^{\prime}(0)=0$ and that $C^{\prime}$ is strictly convex.

1. There exist two numbers $\underline{w}_{1}$ and $\bar{w}_{1}$ with $\underline{w}_{1}<\bar{w}_{1}$, such that:

(a) for $w_{1}>\bar{w}_{1}, \varsigma^{*}\left(w_{1}, \underline{\theta}\right)=v(\underline{y})$, for $w_{1}<\bar{w}_{1}, \varsigma_{1}^{*}\left(w_{1}, \underline{\theta}\right)=v(\bar{y})$.

(b) for $w_{1}>\underline{w}_{1}, \varsigma^{*}\left(w_{1}, \bar{\theta}\right)=v(\underline{y})$, for $w_{1}<\underline{w}_{1}, \varsigma_{1}^{*}\left(w_{1}, \bar{\theta}\right)=v(\bar{y})$.

2. $B_{1}$ is piecewise concave and differentiable except at $\underline{w}_{1}$ and $\bar{w}_{1}$.

3. $\omega^{*}$ is increasing in $w_{0}$. There exist two numbers $\underline{w}_{0}$ and $\bar{w}_{0}$ with $\underline{w}_{0} \leq \bar{w}_{0}$, such that:

(a) for $w_{0}>\bar{w}_{0}, \omega^{*}\left(w_{0}\right)>\bar{w}_{1}$.

(b) for $w_{0}<\underline{w}_{0}, \omega^{*}\left(w_{0}\right)<\underline{w}_{1}$.

(c) for $w_{0} \in\left(\underline{w}_{0}, \bar{w}_{0}\right), \omega^{*}\left(w_{0}\right) \in\left(\underline{w}_{1}, \bar{w}_{1}\right)$.

\footnotetext{
${ }^{16}$ We do not impose a lower bound on continuation utility for this example. We discuss the implications of this bound below.
} 
In our first example, we specialise this environment further by assuming that the initial distribution over utility promises is degenerate at $\widehat{w}_{0} \in\left(\underline{w}_{0}, \bar{w}_{0}\right)$. Consequently, we treat $\varphi_{0}^{*}$ and $\omega^{*}$ as numbers. The assumption that $\widehat{w}_{0} \in\left(\underline{w}_{0}, \bar{w}_{0}\right)$ ensures that the problem is interesting, since then $\varsigma_{1}^{*}\left(\omega^{*}, \underline{\theta}\right)=v(\bar{y})$ and $\varsigma_{1}^{*}\left(\omega^{*}, \bar{\theta}\right)=v(\underline{y})$. In this case, standard arguments establish that the incentive-compatibility constraint binds for the $\underline{\theta}$ type, so that:

$$
\varphi_{1}^{*}\left(\omega^{*}, \underline{\theta}\right)-\varphi_{1}^{*}\left(\omega^{*}, \bar{\theta}\right)=\underline{\theta}\left[\varsigma_{1}^{*}\left(\omega^{*}, \bar{\theta}\right)-\varsigma_{1}^{*}\left(\omega^{*}, \underline{\theta}\right)\right]>0
$$

Let $a^{*}=\left\{c_{0}^{*}, c_{1}^{*}, y_{1}^{*}, B_{1}^{*}\right\}$ denote the associated constrained efficient resource allocation rule. In this example, since consumption and the continuation planner cost are identical across agents in period 0 and since consumption and labour in period 1 differ across agents only to the extent that agents receive different values of the shock, we supress the dependence of these variables on the current planner cost. Thus, we let $c_{0}^{*}=C\left(\varphi_{0}^{*}\right), B_{1}^{*}=B_{1}\left(\omega^{*}\right), y_{1}^{*}(\theta)=Y\left(\varsigma_{1}^{*}\left(\omega^{*}, \theta\right)\right)$ and $c_{1}^{*}(\theta)=C\left(\varphi_{1}^{*}\left(\omega^{*}, \theta\right)\right)$. Using the notation from Remark $2, \mathcal{B}_{1}^{*}=\left\{B_{1}^{*}\right\}$ and, by assumption, $\mathcal{Y}_{1}^{*}\left(b_{1}^{*}\right)=\mathcal{Y}=\{\underline{y}, \bar{y}\}$. The first order conditions for $\varphi_{0}^{*}, \varphi_{1}^{*}, \omega^{*}$ and the period 1 envelope condition imply the inverted Euler equation:

$$
\frac{1}{u^{\prime}\left(c_{0}^{*}\right)}=\frac{q}{\beta}\left[\frac{\pi(\bar{\theta})}{u^{\prime}\left(c_{1}^{*}(\bar{\theta})\right)}+\frac{\pi(\underline{\theta})}{u^{\prime}\left(c_{1}^{*}(\underline{\theta})\right)}\right] .
$$

Implementation. In the corresponding market economy, agents are endowed with $b_{0}=B_{0}\left(\widehat{w}_{0}\right)$ claims in period 0 . They allocate this wealth between current consumption $c_{0}$ and claims $b_{1}$ that trade at price $q$. They do not pay taxes in period 0 . In period 1 , they receive the preference shock, supply labour, pay taxes and consume. The tax function is given by $T\left(b_{1}, y_{1}\right)$. The agent's problem is:

$$
\begin{array}{ll}
V_{0}\left(b_{0}\right)= & \sup _{\left\{c_{0}, c_{1}, y_{1}, b_{1}\right\}} u\left(c_{0}\right)+\beta E\left[u\left(c_{1}(\theta)\right)+\theta v\left(y_{1}(\theta)\right)\right] \\
\text { s.t. } & b_{0}=c_{0}+q b_{1} \\
& \forall \theta: b_{1}=c_{1}(\theta)+T\left(b_{1}, y_{1}(\theta)\right)-y_{1}(\theta) .
\end{array}
$$

We denote the solution to the agents' problem in the market economy by: $\widehat{a}=\left\{\widehat{c}_{0}, \widehat{c}_{1}, \widehat{y}_{1}, \widehat{b}_{1}\right\}$.

To implement the constrained efficient allocation, the government selects $T\left(b_{1}, y_{1}\right)$ to ensure $\widehat{a}=a^{*}$. Given our setting of the initial wealth levels and the absence of taxation in period 0 , to guarantee that the constrained efficient allocation is affordable, the tax system must satisfy:

$$
T\left(B_{1}^{*}, y_{1}^{*}(\theta)\right)=B_{1}^{*}+y_{1}^{*}-c_{1}^{*}(\theta)
$$


Incentive-compatibility of the constrained efficient allocation then immediately guarantees that if the agent saves $B_{1}^{*}$, she will choose the constrained efficient labour supply:

$$
\begin{aligned}
& \bar{y} \in \arg \max _{y \in\{\underline{y}, \bar{y}\}} u\left(B_{1}^{*}-T\left(B_{1}^{*}, y\right)+y\right)+\underline{\theta} v(y), \\
& \underline{y} \in \arg \max _{y \in\{\underline{y}, \bar{y}\}} u\left(B_{1}^{*}-T\left(B_{1}^{*}, y\right)+y\right)+\bar{\theta} v(y) .
\end{aligned}
$$

Using (44), the constrained efficient allocation pins down the tax function on $\mathcal{B}_{1}^{*}=\left\{B_{1}^{*}\right\}$. However, agents in the market economy can choose savings levels in period 0 that are different from $B_{1}^{*}$. Our tax function must be defined for these as well. Since these savings levels represent and reveal deviations from constrained efficient behaviour, the tax function must be set so as to discourage them. One way to do this, following the proof of Proposition 1, is to use the entire constrained efficient allocation rule to set taxes at all possible period 1 wealth levels. However, as Remark 2 indicates there are other ways to discourage such savings choices. One simple approach is to use tax functions that are linear in wealth and of the form:

$$
T(b, y)=T_{0}(y)+T_{1}(y) b .
$$

We show in this section that there is a unique tax function, $\widehat{T}(b, y)=\widehat{T}_{0}(y)+\widehat{T}_{1}(y) b$, within this class that implements the constrained efficient allocation. Moreover, we also show that all optimal tax functions differentiable in wealth satisfy $\frac{\partial T}{\partial b}\left(B_{1}^{*}, y\right)=\widehat{T}_{1}(y)$. Thus, the constrained efficient allocation determines both the tax level and the marginal asset tax at $B_{1}^{*}$.

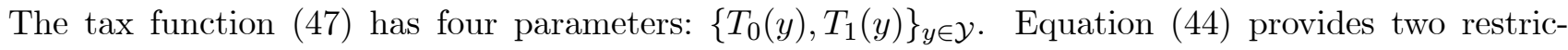
tions on these parameters. The agent's Euler equation must also be satisfied at the constrained efficient allocation:

$$
q u^{\prime}\left(c_{0}^{*}\right)=\beta E\left[\left(1-T_{1}\left(y_{1}^{*}\right)\right) u^{\prime}\left(c_{1}^{*}\right)\right] .
$$

This provides a third restriction. At first sight the restrictions (44), (47) and (48) seem to be sufficient to determine an optimal tax system. If a tax system satisfies them, then the constrained-efficient allocation satisfies the agent's optimality conditions (45), (46) and (48). However, we show that this conclusion is false. We do so by presenting a tax function that satisfies the conditions (44), (47) and (48) but fails to implement the constrained efficient allocation. This tax function is the simplest and most natural candidate for generating the intertemporal wedge: it is separable in wealth and labour supply and the marginal asset tax does not depend on labor. It fails because it admits a profitable deviation in which the agent saves more than $B_{1}^{*}$ in period 0 and works too little in period 1 . 
Lemma $5 a^{*}$ cannot be implemented in a market economy with a tax function of the form $T_{0}(y)+T_{1} b . A$ labour-contingent marginal asset tax is necessary for implementation.

Proof: Assume instead that $a^{*}$ can be implemented with a tax function of the form $T_{0}(y)+T_{1} b$. Then, (44) and (48) require that $T(b, y)=\widehat{T}(b, y): \equiv \widehat{T}_{0}(y)+\widehat{T}_{1} b$, where

$$
\begin{gathered}
\widehat{T}_{1}=\frac{q u^{\prime}\left(c_{0}^{*}\right)-\beta E\left[u^{\prime}\left(c_{1}^{*}\right)\right]}{\beta E u^{\prime}\left(c_{1}^{*}\right)}, \\
\widehat{T}_{0}(\underline{y})=\left(1-\widehat{T}_{1}\right) B_{1}^{*}-c_{1}^{*}(\bar{\theta})+\underline{y} \text { and } \widehat{T}_{0}(\bar{y})=\left(1-\widehat{T}_{1}\right) B_{1}^{*}-c_{1}^{*}(\underline{\theta})+\bar{y} .
\end{gathered}
$$

The binding incentive-compatibility constraint (42) in the component planner's problem implies that an agent can obtain a lifetime expected utility equal to that from $a^{*}$ by choosing an alternative allocation in which she saves $B_{1}^{*}$, and selects $\underline{y}$ and $c_{1}^{*}(\bar{\theta})$ in all states in period 1 . The binding incentive compatibility constraint also implies $c_{1}^{*}(\bar{\theta})<c_{1}^{*}(\underline{\theta})$. It follows that:

$$
q u^{\prime}\left(c_{0}^{*}\right)=\beta\left(1-\widehat{T}_{1}\right) E\left[u^{\prime}\left(c_{1}^{*}\right)\right]<\beta\left(1-\widehat{T}_{1}\right) u^{\prime}\left(c_{1}^{*}(\bar{\theta})\right) .
$$

Thus, $a^{*}$ is dominated by an allocation in which the agent saves $B_{1}^{*}+\varepsilon$ (for $\varepsilon>0$ and small) and chooses $\underline{y}$ regardless of her shock. But this contradicts the optimality of $a^{*}$ for the agent in the market economy. Thus, the tax function $\widehat{T}$ fails to implement $a^{*}$ and a labour-contingent marginal asset tax is necessary for implementation.

In Lemmas 6 and 7 we identify and characterise the unique tax function within the class (47) that does implement the constrained-efficient allocation in the market economy. The crucial property of this tax function is that the marginal asset tax depends on labour earnings ${ }^{17}$. In particular, it is set to equate the marginal value of an extra unit of savings across all states:

$$
\begin{aligned}
& q u^{\prime}\left(c_{0}^{*}\right)=\beta\left(1-T_{1}(\underline{y})\right) u^{\prime}\left(c_{1}^{*}(\bar{\theta})\right), \\
& q u^{\prime}\left(c_{0}^{*}\right)=\beta\left(1-T_{1}(\bar{y})\right) u^{\prime}\left(c_{1}^{*}(\underline{\theta})\right) .
\end{aligned}
$$

Thus, the agent does not find it profitable to increase savings at time 0 irrespective of her choice of labour at time 1. This marginal asset tax has a negative covariance with consumption and is zero on average.

\footnotetext{
${ }^{17}$ This finding is related to Golosov and Tsyvinski (2003), who consider the design of optimal disability insurance. They show that disability benefits must be made contingent on an age dependent asset level.
} 
Lemma 6 Assume that all agents have initial wealth $b_{0}=B_{0}\left(\widehat{w}_{0}\right)$ and that there are no taxes levied in period 0. Define

$$
\begin{aligned}
& T_{1}(\bar{y})=\bar{T}_{1}=1-\frac{q}{\beta} \frac{u^{\prime}\left(c_{0}^{*}\right)}{u^{\prime}\left(c_{1}^{*}(\underline{\theta})\right)}, \\
& T_{1}(\underline{y})=\underline{T}_{1}=1-\frac{q}{\beta} \frac{u^{\prime}\left(c_{0}^{*}\right)}{u^{\prime}\left(c_{1}^{*}(\bar{\theta})\right)},
\end{aligned}
$$

and

$$
\begin{aligned}
& \bar{T}_{0}=\left(1-\bar{T}_{1}\right) B_{1}^{*}+\bar{y}-c_{1}^{*}(\underline{\theta}), \\
& \underline{T}_{0}=\left(1-\underline{T}_{1}\right) B_{1}^{*}+\underline{y}-c_{1}^{*}(\bar{\theta}) .
\end{aligned}
$$

Then the tax function

$$
\begin{aligned}
T(\bar{y}, b) & =\bar{T}_{0}+\bar{T}_{1} b \\
T(\underline{y}, b) & =\underline{T}_{0}+\underline{T}_{1} b
\end{aligned}
$$

implements the allocation $a^{*}$ in a market economy with taxes. Additionally, this is the unique tax function in the class $T(b, y)=T_{0}(y)+T_{1}(y) b$ that does so.

Proof: See Appendix.

Lemma 7 The tax function (54) satisfies:

1. $\underline{T}_{1}>\bar{T}_{1}$.

2. $\underline{T}_{1} \pi(\bar{\theta})+\bar{T}_{1} \pi(\underline{\theta})=0$.

Proof: The first condition follows from the definitions of $\underline{T}_{1}$ and $\bar{T}_{1}$ and the fact that $c_{1}^{*}(\bar{\theta})<c_{1}^{*}(\underline{\theta})$. For the second condition, combine the definitions of $\underline{T}_{1}$ and $\bar{T}_{1}$ with the component planner's intertemporal first order condition (43) to obtain

$$
\underline{T}_{1} \pi(\bar{\theta})+\bar{T}_{1} \pi(\underline{\theta})=1-\frac{q}{\beta} u^{\prime}\left(c_{0}^{*}\right)\left\{\frac{\pi(\bar{\theta})}{u^{\prime}\left(c_{1}^{*}(\bar{\theta})\right)}+\frac{\pi(\underline{\theta})}{u^{\prime}\left(c_{1}^{*}(\underline{\theta})\right)}\right\}=0 .
$$

Although the results in Lemmas 6 and 7 have been derived for a linear wealth tax, similar arguments hold for any tax function differentiable in wealth. Formally, we have the following result. 
Lemma 8 Assume that all agents have initial wealth $b_{0}=B_{0}\left(\widehat{w}_{0}\right)$ and that there are no taxes levied in period 0. Suppose that $T(b, y)$ implements $a^{*}$ and that it is differentiable in $b$, then

$$
\begin{aligned}
& \frac{\partial T}{\partial b}\left(B_{1}^{*}, \bar{y}\right)=\bar{T}_{1}=1-\frac{q}{\beta} \frac{u^{\prime}\left(c_{0}^{*}\right)}{u^{\prime}\left(c_{1}^{*}(\underline{\theta})\right)}, \\
& \frac{\partial T}{\partial b}\left(B_{1}^{*}, \underline{y}\right)=\underline{T}_{1}=1-\frac{q}{\beta} \frac{u^{\prime}\left(c_{0}^{*}\right)}{u^{\prime}\left(c_{1}^{*}(\bar{\theta})\right)},
\end{aligned}
$$

and

$$
E\left[\frac{\partial T}{\partial b}\left(B_{1}^{*}, y\right)\right]=0
$$

The proof is almost identical to that used to obtain the second part of Lemma 6 and Lemma 7 and is omitted.

\subsection{Example 2: More Zero Expected Marginal Asset Taxes}

Component planner's problem We generalise the previous example to allow for heterogeneity in agents' initial utility promises. The component planner's problem the same as in Example 1, except that now $\mathcal{W}_{0}=\left(\underline{w}_{0}, \bar{w}_{0}\right)$. This initial heterogeneity in utility promises results in differences in consumption across agents in the constrained efficient allocation. However, since we restrict attention to $w_{0} \in\left(\underline{w}_{0}, \bar{w}_{0}\right)$, as in Example 1, an agent's period 1 constrained efficient labour allocation does not depend on an agent's initial utility promise. Let $\alpha^{*}=\left\{c_{0}^{*}, c_{1}^{*}, y_{1}^{*}, B_{1}^{*}\right\}$ denote the constrained efficient resource allocation rule for this problem, where these functions are defined as in Lemma 3. For this problem, $\mathcal{B}_{1}^{*}=\left(B_{1}\left(\underline{w}_{1}\right), B_{1}\left(\bar{w}_{1}\right)\right)$, while Graph $\mathcal{Y}_{1}^{*}=\mathcal{B}_{1}^{*} \times \mathcal{Y}$.

Implementation We set agents' initial wealth in the market economy according to: $b_{0}\left(w_{0}\right)=B_{0}\left(w_{0}\right)$. Once again this setting of initial wealths, coupled with the absence of taxes in period 0, implies that an agent with initial wealth $b_{0}$ must be induced to save $B_{1}^{*}\left(b_{0}\right) \in \mathcal{B}_{1}^{*}$. Affordability of the period 1 allocation then pins down taxes on Graph $\mathcal{Y}_{1}^{*}$ according to:

$$
T\left(b_{1}, y_{1}^{*}\left(b_{1}, \theta\right)\right)=b_{1}+y\left(b_{1}, \theta\right)-c_{1}^{*}\left(b_{1}, \theta\right)
$$

Since $\mathcal{B}_{1}^{*}$ is a larger set in this example relative to the last, this condition determines the tax function across a broader range of wealth levels. As before deviations to savings levels outside of $\mathcal{B}_{1}^{*}$ are inconsistent with constrained efficient behaviour and such deviations need to be discouraged. Lemma 8 suggests that if $T$ is differentiable then, in order to rule out joint deviations, it will need to satisfy the state-by-state Euler 
equations (55) at each $b_{1} \in \mathcal{B}_{1}^{*}$. We now show that the planner's optimality conditions directly imply that $T$ satisfies $(55)$ on $\mathcal{B}_{1}^{*}$.

Since $\mathcal{W}_{0}=\left(\underline{w}_{0}, \bar{w}_{0}\right)$, the constrained efficient labor allocation does not depend on promised utility. It then follows from the component planner's period 1 incentive and promise keeping constraints that $w_{1}=\varphi_{1}^{*}\left(w_{1}, \bar{\theta}\right)+K(\bar{\theta})=\varphi_{1}^{*}\left(w_{1}, \underline{\theta}\right)+K(\underline{\theta})$, for $w_{1} \in \mathcal{W}_{1}^{*}=\left(\underline{w}_{1}, \bar{w}_{1}\right)$, where $K(\bar{\theta})$ and $K(\underline{\theta})$ are constants that do not depend on $w_{1}$. Thus, $\partial \varphi_{1}^{*}\left(w_{1}, \theta\right) / \partial w_{1}=1$ for each $\theta$ on $\mathcal{W}_{1}^{*}$. Substituting $B_{1}\left(w_{1}\right)$ for $b_{1}$ in (56), totally differentiating with respect to $w_{1}$ and using $c_{1}^{*}\left(B_{1}\left(w_{1}\right), \theta\right)=C\left(\varphi_{1}^{*}\left(w_{1}, \theta\right)\right)$, it follows that

$$
\frac{\partial T}{\partial b}\left(B_{1}\left(w_{1}\right), \bar{y}\right)=1-C^{\prime}\left(\varphi_{1}^{*}\left(w_{1}, \underline{\theta}\right)\right) \frac{1}{B_{1}^{\prime}\left(w_{1}\right)},
$$

for $w_{1} \in\left(\underline{w}_{1}, \bar{w}_{1}\right)$. Then, using the component planner's period 0 first order condition, we have:

$$
C^{\prime}\left(\varphi_{1}^{*}\left(\omega^{*}\left(w_{0}\right), \underline{\theta}\right)\right) \frac{1}{B_{1}^{\prime}\left(\omega^{*}\left(w_{0}\right)\right)}=\frac{q}{\beta} \frac{u^{\prime}\left(c_{0}^{*}\left(b_{0}\right)\right)}{u^{\prime}\left(c_{1}^{*}\left(B_{1}^{*}\left(b_{0}\right), \underline{\theta}\right)\right)} .
$$

Combining (57) and (58) for $\theta=\underline{\theta}$, and applying identical reasoning to $\theta=\bar{\theta}$, we have:

$$
\begin{aligned}
& q u^{\prime}\left(c_{0}^{*}\left(b_{0}\right)\right)=\beta\left(1-\frac{\partial T}{\partial b}\left(B_{1}^{*}\left(b_{0}\right), \underline{y}\right)\right) u^{\prime}\left(c_{1}^{*}\left(B_{1}^{*}\left(b_{0}\right), \underline{y}\right)\right), \\
& q u^{\prime}\left(c_{0}^{*}\left(b_{0}\right)\right)=\beta\left(1-\frac{\partial T}{\partial b}\left(B_{1}^{*}\left(b_{0}\right), \bar{y}\right)\right) u^{\prime}\left(c_{1}^{*}\left(B_{1}^{*}\left(b_{0}\right), \bar{y}\right)\right) .
\end{aligned}
$$

Thus, the state-by-state Euler equations hold for $w_{0} \in\left(\underline{w}_{0}, \bar{w}_{0}\right)$. It follows that the expected marginal asset tax, $E_{\theta}\left[\frac{\partial T\left(b_{1}, y_{1}^{*}\left(b_{1}, \theta\right)\right)}{\partial b}\right], \forall b_{1} \in \mathcal{B}_{1}^{*}$, is zero. The argument is identical to the proof of Lemma 6 .

This example provides an illustration of the more general result obtained in the proof of Proposition 1. Simply setting taxes so that agents are induced to choose the constrained efficient consumption and labour allocation given their wealth level at time 1 is enough to rule out joint labour and savings deviations. Here, this implies that the Euler equation holds state by state at the appropriate wealth level, leading to a zero expected marginal asset tax. This example, however, is special in that the first period constrained efficient labour allocation does not depend on the agent's continuation utility promise. Equivalently, in the market economy, the agent's labour supply in each $\theta$ state is independent of $b_{1} \in \mathcal{B}_{1}^{*}$. More generally, this is not true. Agent's with different period 1 utility promises or wealth levels will be entitled to different labour supply allocations. In the next example, we show that when this happens the expected marginal asset tax will not be zero. 


\subsection{Example 3: Positive Expected Marginal Asset Taxes}

We extend the previous example to a continuous support for the preference shock in period 1, so that $\Theta \in[\underline{\theta}, \bar{\theta}]$. We assume that $\pi$ admits a strictly positive density $\rho$. This extension implies that the constrained efficient labour allocation in period 1 varies across agents with different initial promised utilities. The initial period of the component planner's problem is as before. In period 1, the component planner solves:

$$
\begin{array}{ll}
B_{1}\left(w_{1}\right)= & \inf _{\varphi: \Theta \rightarrow \mathcal{U}, \varsigma: \Theta \rightarrow\{\underline{v}, \bar{v}\}} \int_{\underline{\theta}}^{\bar{\theta}}\{C(\varphi(\theta))-Y(\varsigma(\theta))\} d \pi \\
\text { s.t. } & w_{1}=\int_{\underline{\theta}}^{\bar{\theta}}\{\varphi(\theta)+\theta \varsigma(\theta)\} d \pi, \\
& \forall \theta, \theta^{\prime}, \quad \varphi(\theta)+\theta \varsigma(\theta) \geq \varphi\left(\theta^{\prime}\right)+\theta \varsigma\left(\theta^{\prime}\right) .
\end{array}
$$

The incentive-compatibility constraints imply that if $\varsigma(\theta)=\varsigma\left(\theta^{\prime}\right)$, then $\varphi(\theta)=\varphi\left(\theta^{\prime}\right)$ for any $\theta, \theta^{\prime} \in \Theta$. They also imply that $\varsigma$ must be monotone. Thus, the component planning problem in period 1 can be rewritten as:

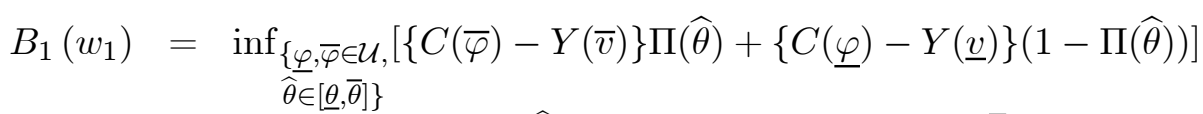

$$
\begin{aligned}
& \text { s.t. } \quad w_{1}=\left\{\bar{\varphi} \Pi[\widehat{\theta}]+\bar{v} \int_{\underline{\theta}}^{\widehat{\theta}} \theta d \pi\right\}+\left\{\underline{\varphi}(1-\Pi[\widehat{\theta}])+\underline{v} \int_{\widehat{\theta}}^{\bar{\theta}} \theta d \pi\right\} \text {, } \\
& \bar{\varphi}+\widehat{\theta} \bar{v}=\underline{\varphi}+\widehat{\theta} \underline{v} \text {, }
\end{aligned}
$$

where $\Pi[\widehat{\theta}]=\int_{\underline{\theta}}^{\widehat{\theta}} d \pi$ and $\underline{v}=v(\underline{y})$ and $\bar{v}=v(\bar{y})$. In this problem, the component planner chooses a cut off value for shocks $\widehat{\theta}$ and a utility allocation of the form: $\{\varphi(\theta), \varsigma(\theta)\}=\{\underline{\varphi}, \underline{v}\}, \theta<\hat{\theta}$ and $\{\varphi(\theta), \varsigma(\theta)\}=\{\bar{\varphi}, \bar{v}\}$, $\theta \geq \hat{\theta}$. Let $\left\{\underline{\varphi}_{1}^{*}, \bar{\varphi}_{1}^{*}, \widehat{\theta}_{1}^{*}\right\}$ denote optimal choices of $\underline{\varphi}, \bar{\varphi}$ and $\hat{\theta}$ as functions of the component planner's period 1 utility promise $w_{1} \cdot{ }^{18}$ We modify the notation for a constrained efficient resource allocation rule accordingly and, for $b_{1} \in \mathcal{B}_{1}^{*}$, let $\underline{c}_{1}^{*}\left(b_{1}\right)=C\left(\underline{\varphi}_{1}^{*}\left(B_{1}^{-1}\left(b_{1}\right)\right)\right), \bar{c}_{1}^{*}\left(b_{1}\right)=C\left(\bar{\varphi}_{1}^{*}\left(B_{1}^{-1}\left(b_{1}\right)\right)\right)$ and $\theta_{1}^{*}\left(b_{1}\right)=\widehat{\theta}_{1}^{*}\left(B_{1}^{-1}\left(b_{1}\right)\right)$. As before, we construct the optimal tax function on $\mathcal{B}_{1}^{*}$ so that the constrained efficient allocation is affordable:

$$
\begin{aligned}
& T\left(b_{1}, \bar{y}\right)=b_{1}+\bar{y}-\underline{c}_{1}^{*}\left(b_{1}\right) \\
& T\left(b_{1}, \underline{y}\right)=b_{1}+\underline{y}-\bar{c}_{1}^{*}\left(b_{1}\right) .
\end{aligned}
$$

The function $\theta_{1}^{*}$ summarises the effect of different wealth levels in $\mathcal{B}_{1}^{*}$ upon the agent's first period labour allocation. We will refer to the dependence of $\theta_{1}^{*}$ on $b_{1}$ as a wealth effect. Lemma 9 provides a sufficient condition for $\theta_{1}^{*}$ to be non-constant over all $b_{1} \in \mathcal{B}_{1}^{*}$.

\footnotetext{
${ }^{18}$ If these choices are not unique, we take a selection from the component planner's optimal policy correspondence.
} 
Lemma 9 If $C^{\prime \prime} / C^{\prime}$ is non-decreasing and $b_{1}$ and $b_{1}^{\prime}$ are in $\mathcal{B}_{1}^{*}$, then $\widehat{\theta}_{1}^{*}\left(b_{1}\right) \neq \widehat{\theta}_{1}^{*}\left(b_{1}\right)$.

\section{Proof: See Appendix.}

Remark 4: If the agent's utility function $u$ is CARA or CRRA with coefficient of relative risk aversion greater than or equal to 1 , then $C^{\prime \prime} / C^{\prime}$ is non-decreasing.

We now show that the presence of wealth effects on the labour allocation implies that the expected marginal asset tax is not zero. This a straightforward implication of two properties of the tax system. First, as observed in Remark 3, an agent's expected taxes in each period are zero provided she selects the prescribed labour allocation. Second, the agent pays higher taxes at all wealth levels in the high labour income state.

To derive implications for marginal asset taxes, first note that by the first property, for each $b_{1} \in \mathcal{B}_{1}^{*}$

$$
\begin{aligned}
& T\left(b_{1}, \bar{y}\right) \Pi\left[\theta_{1}^{*}\left(b_{1}\right)\right]+T\left(b_{1}, \underline{y}\right)\left(1-\Pi\left[\theta_{1}^{*}\left(b_{1}\right)\right]\right) \\
& \quad=\left(b_{1}+\bar{y}-\bar{c}_{1}^{*}\left(b_{1}\right)\right) \Pi\left[\theta_{1}^{*}\left(b_{1}\right)\right]+\left(b_{1}+\underline{y}-\underline{c}_{1}^{*}\left(b_{1}\right)\right)\left(1-\Pi\left[\theta_{1}^{*}\left(b_{1}\right)\right]\right)=0 .
\end{aligned}
$$

Next consider an agent who increases her savings from $b_{1}^{*} \in \mathcal{B}_{1}^{*}$ to $b_{1}^{*}+\delta \in \mathcal{B}_{1}^{*}$, but keeps her labour allocation the same. Then, the change in the agent's expected tax payment is given by

$$
\begin{aligned}
\Delta T & \equiv E\left[T\left(b_{1}+\delta, y_{1}^{*}\left(b_{1}, \theta\right)\right)-T\left(b_{1}, y_{1}^{*}\left(b_{1}, \theta\right)\right)\right] \\
& =E\left[T\left(b_{1}+\delta, y_{1}^{*}\left(b_{1}, \theta\right)\right)-T\left(b_{1}+\delta, y_{1}^{*}\left(b_{1}+\delta, \theta\right)\right)\right] \\
& =\left(T\left(b_{1}+\delta, \bar{y}\right)-T\left(b_{1}+\delta, \underline{y}\right)\right)\left(\Pi\left[\theta_{1}^{*}\left(b_{1}\right)\right]-\Pi\left[\theta_{1}^{*}\left(b_{1}+\delta\right)\right],\right.
\end{aligned}
$$

where the first equality follows from (61). It follows that since $T\left(b_{1}+\delta, \bar{y}\right)>T\left(b_{1}+\delta, \underline{y}\right)$, the sign of $\Delta T$ depends on $\Delta \Pi \equiv \Pi\left[\theta_{1}^{*}\left(b_{1}\right)\right]-\Pi\left[\theta_{1}^{*}\left(b_{1}+\delta\right)\right]$. This term is a function of the effect of first period wealth on the labor allocation. By Lemma 9 and the fact that $\rho(\theta)>0$, when $C^{\prime \prime} / C^{\prime}$ is non-decreasing, $\Delta \Pi$ is always non-zero and, hence, the agent's tax payment changes if she alters her savings level without correspondingly altering her labour supply. $\Delta T / \delta$ provides a discrete approximation to an agent's marginal asset tax. ${ }^{19}$ It follows that this too is linked to the presence wealth effects on labour supply and, under the condition of Lemma 9, is non-zero.

\footnotetext{
${ }^{19}$ If $\theta^{*}$ is differentiable and $\mathcal{B}_{1}^{*}$ is an interval then the differential version of $(62)$ is $E\left[\frac{\partial T}{\partial b}(b, y)\right]=-(T(b, \bar{y})-T(b, \underline{y})) \rho\left(\theta^{*}(b)\right)$ $\frac{\partial \theta^{*}}{\partial b}$, where $\frac{\partial \theta^{*}}{\partial b}$ is the wealth effect term.
} 
The presence of wealth effects on labor supply also implies that a tax system of the form $T\left(b_{1}, y_{1}\right)$ that satisfies the state-by-state Euler equation cannot implement the constrained efficient allocation. To see this, define $F\left(b_{1}, y_{1}\right): \equiv \beta u\left(b_{1}+y-T\left(b_{1}, y\right)\right)$. This function represents the discounted utility from affordable consumption at time 1 under the tax system. Assuming $F$ is differentiable in $b_{1}$, a tax function $T$ set so that the state-by-state Euler equation holds implies that $\partial F / \partial b_{1}$ is independent of $y_{1}$, so that $F$ is additively separable in $b_{1}$ and $y_{1}$ and of the form: $F\left(b_{1}, y_{1}\right)=R\left(b_{1}\right)+S\left(y_{1}\right)$. Then, an agent choosing labour in the market economy would maximise $S(y)+\theta v(y)$, and the optimal choice of labour by the agent would be independent of wealth. Thus, a tax function that satisfies the state-by-state Euler equations cannot be used to implement a wealth-dependent labour allocation.

It is important to emphasise that this does not mean that the marginal asset tax will be non-stochastic and independent of an agent's labour. (60) will still imply that the marginal tax on assets will be different at low and high labor supplies at a given wealth level. Absent such a feature, the tax system would be unable to rule out joint deviations analogous to those in our first example, in which an agent with initial wealth $b_{0}$ saves more than $B_{1}^{*}\left(b_{0}\right)$ and chooses $\underline{y}$ for some $\theta<\theta^{*}\left(B_{1}^{*}\left(b_{0}\right)\right)$. Instead in this example, and in general, the government discourages saving by both reducing the after-tax expected return from saving and increasing the covariance of that return with labour earnings. To analyse these effects further, we study numerical examples in section 7.

\subsection{Utility bounds and borrowing limits}

The previous examples focus on the implications of the incentive compatibility constraint for the tax system. The lower bound on the continuation utilities in the component planning problems also has implications both for taxes and the structure of asset markets. The argument in the proof of Proposition 1 implies that this lower bound can be implemented with a lower bound on an agent's claim holdings. In our implementation, an agent with utility promise $w_{t}$ and shock $\theta$ is induced to save $B_{t+1}\left(\omega_{t}^{*}\left(w_{t}, \theta\right)\right)$, the corresponding continuation cost of a component planner. If the component planner is restricted to making utility promises in excess of $\underline{U}_{t+1}$, then our implementation will require agents to hold claims in excess of $\underline{b}_{t+1}: \equiv B_{1}\left(\underline{U}_{t+1}\right)$. This borrowing limit will bind on those agents with low after-tax resources, $x_{t}=b_{t}+y_{t}-T_{t}\left(b_{t}, y_{t}\right)$, in period $t$. When it binds, it also generates a wedge in the intertemporal Euler equations of agents, though one that runs in the opposite direction to the wedge generated by the tax 
system. Specifically, the agent's Euler equation is:

$$
q_{t} u^{\prime}\left(c_{t}\right)=\beta E_{t}\left[\left(1-\frac{\partial T_{t+1}}{\partial b}\left(b_{t+1}, y_{t+1}\right)\right) u^{\prime}\left(c_{t+1}\right)\right]+\widehat{\eta}_{t+1},
$$

where $\widehat{\eta}_{t+1}$ denotes the multiplier on the borrowing limit. Consequently, the intertemporal wedge of an agent can be decomposed as follows:

$$
\underbrace{\frac{\beta E_{t} u^{\prime}\left(c_{t+1}\right)-q_{t} u^{\prime}\left(c_{t}\right)}{\beta E_{t} u^{\prime}\left(c_{t+1}\right)}}_{\text {Intertemporal Wedge }}=\underbrace{\frac{E_{0}\left[\frac{\partial T_{t+1}}{\partial b}\left(b_{t+1}, y_{t+1}\right) u^{\prime}\left(c_{t+1}\right)\right]}{E_{0} u^{\prime}\left(c_{t+1}\right)}}_{\text {Tax component }}-\underbrace{\frac{\widehat{\eta}_{t+1}}{E_{t} u^{\prime}\left(c_{t+1}\right)}}_{\text {Limit Component }} \text {. }
$$

The first component is induced by the tax system, the second by the multiplier on the borrowing limit. Clearly, the second component is only present when the borrowing limit binds. However, the lower utility bound in the planner's problem also has implications for the optimal tax system. Specifically, the bound restricts the planner's ability to use continuation utilities to provide incentives for truthful revelation in the optimal mechanism. Thus, the planner must rely more heavily on variations in current consumption to provide incentives. Close to the lower bound, the constrained efficient allocation will then exhibit greater consumption variability and larger insurance and effort wedges. These characteristics translate into greater curvature of the optimal tax function at wealth levels close to the borrowing limit.

\section{Extension: The $\infty$-period economy}

For this subsection we set $T=\infty$. We assume that $\mathcal{U}$ and $\mathcal{V}$ are compact sets. In this infinite period setting, this assumption guarantees that a utility allocation satisfying the temporary incentive-compatibility constraints (6) also satisfies the incentive-compatibility condition (5). The definition of an equilibrium in a component planning economy is almost identical to that given in Definition 1 . We simply require that $\sum_{t=0}^{\infty} q_{t}<\infty$ and that Conditions 1 and 2 in that definition are replaced by:

$1^{\prime}$ For all $t, B_{t}$ and $B_{t+1}$ satisfy (11). Additionally, each $B_{t} \geq-\bar{y}\left(1+\sum_{t=0}^{\infty} q_{t}\right) .{ }^{20}$

$2^{\prime}$ For all $t,\left\{\varphi_{t}^{*}, \varsigma_{t}^{*}, \omega_{t+1}^{*}\right\}$ attain the infima in the problems (11).

\footnotetext{
${ }^{20}$ This boundedness in conjunction with an argument similar to Theorem 4.14 in Stokey, Lucas and Prescott (1989) guarantees that the sequence of $\left\{B_{t}\right\}_{t=0}^{\infty}$ satisfies the Bellman equation (11).
} 
Similarly, the definition of a competitive equilibrium in a market economy is the same as Definition 2 except for the requirement that $\sum_{t=0}^{\infty} q_{t}<\infty$ and the replacement of Conditions 1 and 2 in that definition with:

$1^{\prime}$ For all $t, V_{t}$ and $V_{t+1}$ satisfy (19). Additionally, each $V_{t}: B_{t} \rightarrow \mathcal{W}_{t}$.

$2^{\prime}$ For all $t,\left\{\widehat{c}_{t}, \widehat{y}_{t}, \widehat{b}_{t+1}\right\}$ attain the infima in the problems (19).

The definition of implementation is identical to that in the finite period case. We now have the following proposition.

Proposition 3 Assume $\mathcal{U}$ and $\mathcal{V}$ are compact. Let $\xi^{C P}=\left\{\left\{q_{t}\right\}_{t=0}^{\infty},\left\{\varphi_{t}^{*}, \varsigma_{t}^{*}, \omega_{t+1}^{*}\right\}_{t=0}^{\infty},\left\{B_{t}\right\}_{t=0}^{\infty},\left\{\Psi_{t+1}\right\}_{t=0}^{\infty}\right\}$ be an equilibrium of a infinite-period component planner economy $\mathcal{E}^{C P}\left(\left\{\underline{U}_{t+1}\right\}_{t=0}^{\infty},\left\{G_{t}\right\}_{t=0}^{\infty}, \Psi_{0}\right)$. Then, the associated constrained efficient utility allocation can be implemented by a competitive equilibrium in a market economy with taxes and borrowing limits.

Proof: See the Appendix.

\section{Numerical Analysis}

To shed further light on the properties of the optimal tax system, we turn to numerical examples. We set parameters according to recent calibrations of Bewley economies with endogenous labour supply. However, our examples are intended to be illustrative rather than a fully calibrated quantitative exercise. For reasons of space, we only report one example in detail below. However, we indicate those properties that are robust across other examples that we have computed.

\subsection{Calibration and numerical procedure}

We adopt the utility function:

$$
u(c, y ; \theta)=\varkappa \frac{c^{1-\sigma}}{1-\sigma}+(1-\varkappa) \frac{(\bar{y}-\theta y)^{1-\gamma}}{1-\gamma}
$$


Here, $\theta$ may be interpreted as a cost of effort $\operatorname{shock}^{21}$. This preference specification is common in macroeconomics. $^{22}$

The numerical parameters for this economy are $\left\{\varkappa, \sigma, \gamma, \bar{y}, \beta, \bar{U}, \Theta, \pi,\left\{G_{t}\right\}_{t=0}^{\infty}\right\}$. For our benchmark case, we follow Heathcote, Storesletten and Violante (2003) in setting the preference parameters $(1-\varkappa) / \varkappa$ to $1.184, \bar{y}$ to $1, \sigma$ to $1.461, \gamma$ to 2.54 . Heathcoate, Storesletten and Violante choose this parameterisation so that their model matches the empirical fraction of time devoted to labour and the wage-hours correlation for the US. It implies a Frisch elasticity of labour supply of 0.3 . In addition, we set $\beta$ to 0.90 . In the benchmark case, we assume that $1 / \theta$ is distributed uniformly on the interval $[0.2,1.2]$. We set $\underline{U}$ to -3.48 , which translates into a borrowing limit of -2.14 . This value of $\underline{U}$ lies between the lifetime utility that an agent would attain if she were at her "natural" borrowing limit" ${ }^{23}$ in a Bewley economy without taxes, which is clearly $-\infty$, and the lifetime utility under autarky without taxes and markets, equal to -2.74 . Government consumption is constant over time and equal to 0.1 in each period, which amounts to approximately $30 \%$ of period aggregate output.

We numerically solve for the steady state of a component planner economy. ${ }^{24}$ In the steady state, the price of one period non-contingent claims is constant at $q$, the component planner's cost function $B$ and optimal policy functions, $\left\{\varphi^{*}, \varsigma^{*}, \omega^{*}\right\}$ are time invariant, and the cross sectional distribution of utility promises, $\Psi$, is a fixed point of the Markov operator implied by $\omega^{*}$. Our algorithm solves the recursive component planner problem using numerical dynamic programming techniques at each intertemporal price. We use the policy functions from this problem to obtain an approximation to the limiting distribution over utility promises. We iterate on the intertemporal price until this distribution is consistent with resource feasibility. The solutions to the component planner problems imply a time invariant tax function $T(b, y)$ on Graph $\mathcal{Y}^{*}$, where $\mathcal{Y}^{*}(b)=\left\{y: y=Y\left(\varsigma^{*}\left(B^{-1}(b), \theta\right)\right), \theta \in \Theta\right\}$.

\footnotetext{
${ }^{21}$ Alternatively, $\theta$ can be interpreted as the reciprocal of a productivity shock. Then, $y$ should be interpreted as the agent's output.

${ }^{22}$ These preferences retain the key property of additive separability in consumption and labour. They drop the inessential property of multiplicative separability in the shock and the utility from labour. They are clearly not bounded. Below, we assume that the tax functions we compute do not admit an infinite sequence of deviations that raise the agents' payoff above their constrained efficient one.

${ }^{23}$ The natural debt limit is the maximal borrowing that an agent can service. Given the bound on the agent's per period output, this borrowing limit is finite, but it translates into a utility bound of $-\infty$.

${ }^{24}$ We do not have a proof of the existence of a steady state in our environment. The numerical policy functions we compute indicate that the Markov process for utility promises possesses an ergodic distribution. As in Atkeson and Lucas (1995) the lower bound on utilities is essential to ensure this.
} 


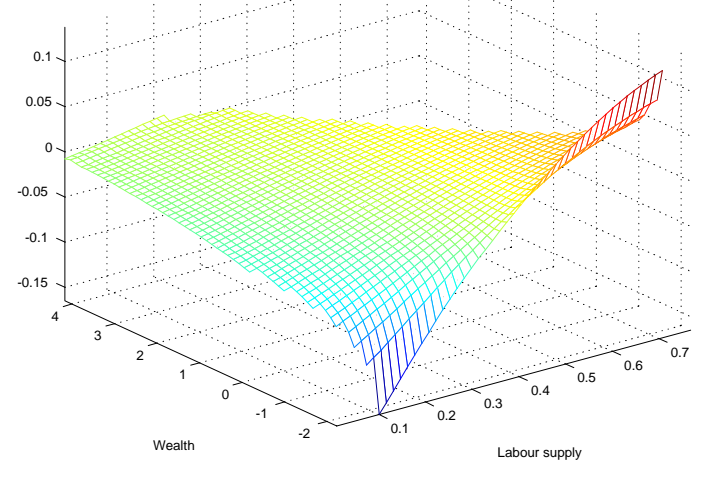

Figure 1: The tax function, $T(b, y)$.

\subsection{Numerical Results}

The optimal tax function $T$ for the benchmark parameterisation is illustrated in Figure 1 on the set Graph $\mathcal{Y}^{*}$. A striking feature of the tax function is the high curvature in the neighbourhood of the borrowing limit. In particular, the cross partial of the tax function is large in absolute value here, making marginal income taxes sensitive to wealth and marginal wealth taxes sensitive to income. This conforms with the discussion in section 5.4. Figure 1 reveals that $T$ is negative for small values of $b$ and $y$, thus agents with low wealths and low incomes receive transfers.

Figure 2 shows $\frac{\partial T(b, y)}{\partial y}$, the marginal labour income tax in our economy, as a function of $y$. Each curve corresponds to a different wealth level $b$. Marginal labour income taxes are decreasing in wealth. They decrease particularly rapidly as wealth levels approach the borrowing limit. We have found this to be a robust feature of optimal tax functions across the various alternative parameterisations that we have computed. In contrast, we have found that the dependence of the marginal labour income tax on labour income is sensitive to the choice of utility function and shock distribution. In the benchmark parameterisation that we illustrate here, marginal labour income taxes have an inverted U shape when drawn as a function of income, holding wealth fixed. At the lowest and highest labour supplies at each wealth level, the marginal income tax is zero. At intermediate levels it is positive. ${ }^{25}$

\footnotetext{
${ }^{25}$ The same pattern has been found in the static non-linear tax literature when similar assumptions on preferences and
} 


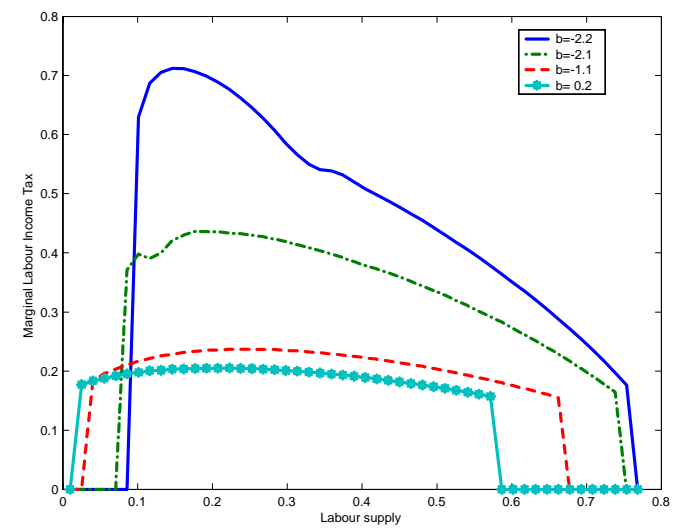

Figure 2: The Marginal Labour Income Tax, $\frac{\partial T(b, y)}{\partial y}$.

It is interesting to compare these findings to those from the static non-linear income taxation literature. That literature has analysed the dependence of optimal marginal income taxes on income only. Given the sensitivity of the optimal tax schedule to the specification of preferences and the underlying shock distribution, few general results are available. In his seminal contribution, Mirrlees (1971) obtained marginal income tax rates that are low and slightly declining in income, while Diamond (1998) and Saez (2001) find marginal income taxes that are high and sharply declining in income at low income levels. ${ }^{26}$ These recent findings have been interpreted as being consistent with the empirical phasing out of social benefits at low incomes. Our result, that marginal income taxes should be high at low wealth levels, complements this. It suggests that any transfers received by low wealth agents should be rapidly phased out as their income rises.

The implications of the intertemporal wedge for marginal asset taxes are illustrated in figures 3 and 4 . Figure 3 plots $\frac{\partial T}{\partial b}(\cdot, y)$ against $b$ for different fixed labour income levels $y$. As the figure indicates, marginal asset taxes vary across incomes. Thus, an agent's period $t+1$ marginal asset tax is stochastic from the shocks are made. The zero marginal income taxes at the lowest and highest labour supplies stem from the fact that the incentive-compatibility constraint does not bind at these points. See Seade (1977).

${ }^{26}$ The low value of marginal income taxes in Mirrlees (1971) stems from his choice of utility function: $\log c+\log (1-l)$, which implies a high labour supply elasticity. The monotonically declining pattern of rates in income stems from his assumption of a log-normal distribution of shocks. Diamond (1998) and Saez (2001) assume lower labour supply elasticities and a (calibrated) Pareto shock distribution, and obtain higher marginal income taxes. 
perspective of period $t$. Moreover, these marginal assets taxes covary negatively with income, being high at low income levels and low at high ones. As with marginal income taxes, variation in marginal asset taxes is greatest close to the borrowing limit. Figure 4 explicitly relates the tax function to the intertemporal wedge. Recall that the contribution of the tax function to the intertemporal wedge can be decomposed into an expected marginal tax and a covariance component:

$$
\frac{E_{t}\left[\frac{\partial T}{\partial b}\left(b_{t+1}, y_{t+1}\right) u^{\prime}\left(c_{t+1}\right)\right]}{E_{t}\left[u^{\prime}\left(c_{t+1}\right)\right]}=E_{t}\left[\frac{\partial T}{\partial b}\left(b_{t+1}, y_{t+1}\right)\right]+\frac{\operatorname{Cov}_{t}\left[\frac{\partial T}{\partial b}\left(b_{t+1}, y_{t+1}\right), u^{\prime}\left(c_{t+1}\right)\right]}{E_{t}\left[u^{\prime}\left(c_{t+1}\right)\right]} .
$$

Figure 4 shows the total contribution of the tax function to the wedge (solid line), as well as the expected marginal asset tax (dashed line) and covariance (dash-dot) components. Since the covariance component is always positive, the total contribution acts as an upper bound for the expected marginal asset tax. The total contribution is small away from the asset limit. Over most of the wealth range it is less than $1 \%$ in value, but close to the borrowing limit it becomes much larger rising to about $16 \%$. The expected marginal asset tax peaks at a little over $2 \%$ at the borrowing limit, and then falls steadily with wealth. The covariance component is also decreasing in wealth, but it is much larger close to the limit and falls off much more quickly as wealth increases. Consequently, the covariance component plays the major role in generating the total contribution of the tax system to the intertemporal wedge only when the agent's wealth is small and the total contribution is high. The small total contribution of the tax function to the intertemporal wedge does not imply that taxation of asset income is small in our economy. The gross return on assets in our benchmark parameterization is approximately equal to $10 \%$. It follows that the corresponding tax on asset income is equal to the tax contribution to the intertemporal wedge multiplied by a factor of $10 .^{27}$

\section{Concluding remarks}

We study optimal taxation in a class of dynamic economies with private information. We show that constrained efficient allocations in this environment can be implemented as competitive equilibria in market

\footnotetext{
${ }^{27}$ The equilibrium value of $q$ is 0.90375 for our benchmark parameterization. The corresponding marginal tax on asset income, $\tau$ can be derived from the agents' intertemporal Euler equation:$$
u^{\prime}\left(c_{t}\right)=\beta E_{t}\left[\left(\frac{1}{q_{t}}-1\right)\left(1-\tau_{t+1}\left(b_{t+1}, y_{t+1}\right)\right)+1\right] u^{\prime}\left(c_{t+1}\right),
$$

evaluated at the constrained efficient allocation.
} 


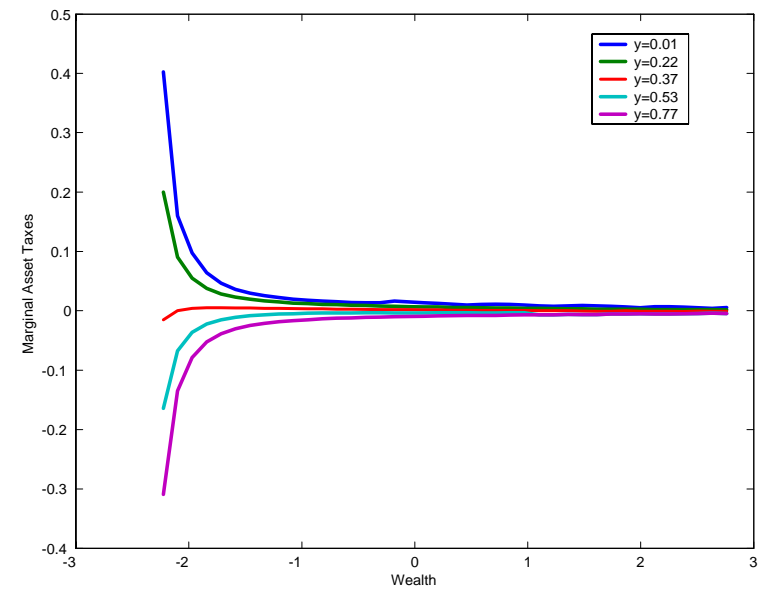

Figure 3: The marginal asset tax, $\frac{\partial T(b, y)}{\partial b}$.

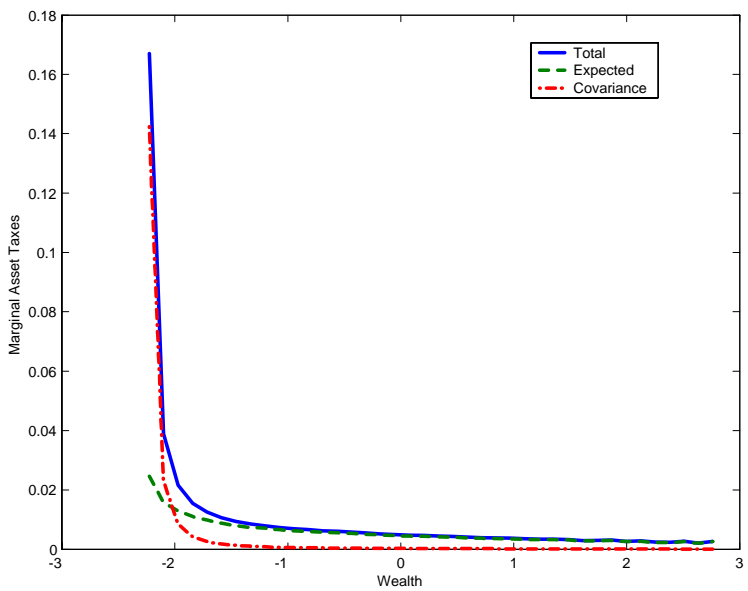

Figure 4: The tax contribution to the intertemporal wedge.

economies with taxes. The optimal tax system is simple and conditions only upon current wealth and current labour earnings. The incentive compatibility constraints shape the features of the resulting optimal tax system. We analytically derive implications for both income and asset taxation and further explore them in numerical examples.

We construct our optimal tax system using the recursive formulation of the planner's problem. This formulation relies on expected utility promises to summarise past histories of shocks. We obtain an agent's constrained efficient continuation allocation as a function of her wealth in the market economy, rather than her expected utility promise. In our fiscal implementation, we then set taxes as functions of an agent's wealth and labour supply so as to ensure that the agent can afford the constrained efficient allocation. These tax functions allow the government to pin down an agent's after-tax resources as a function of her current wealth and current labour supply. We show that given future tax functions, the agent will find it optimal to allocate after-tax resources between consumption and savings in a socially efficient way.

The recursive formulation of the planner's problem that underlies this argument is, however, only valid for the case in which the agent's shocks are i.i.d. Consequently, our fiscal implementation will not work when shocks are persistent. In this case, wealth levels do not adequately describe past histories. Moreover, when shocks are persistent an agent's current shock influences her intertemporal marginal rate of substitution. Thus, the agent's intertemporal allocation of consumption and savings will depend upon 
this shock as well as her after-tax quantity of resources. The simple decomposition of the agent's and component planner's within period problem on which the proof of Proposition 1 relies will no longer holds.

Kocherlakota (2003) provides an alternative fiscal implementation that works even with persistent shocks. In Kocherlakota's formulation, the government keeps track of an agent's entire history of labour supplies and condition taxes upon this history. The government does not use wealth to summarise aspects of an agent's past history.

It remains an open question as to whether there exists a fiscal implementation intermediate between ours and Kocherlakota's that would be valid for an economy with persistent shocks. Doepke and Townsend (2002) and Fernandes and Phelan (2000) have shown that there do exist recursive formulations of the planning problem for Markovian shocks. These rely on a vector of utility promises to keep track of histories. Similarly, a recursive fiscal implementation for an economy with persistent shocks could not rely on an agent's stock of non-contingent claims alone to keep track of past histories. It would be necessary to augment the state space. For example, it may be possible to use an agent's portfolio position in a richer asset market structure in conjunction with truncated labour histories to encode past shock histories. We leave this important extension to future work.

Our fiscal implementation also embeds specific assumptions about the relative roles of markets and government policy. In particular, no private insurance contracts are allowed with the current market structure. In practice, government welfare programs and private credit and insurance contracts are complementary in providing incentives and determining the extent of risk-sharing supported in a competitive equilibrium. Exploring this complementarity could provide important insight in cross-country differences in government policies.

\section{Appendix: Proofs}

Proof of Lemma 2: The constraints in problem (14) are linear and the objective is strictly convex. Hence, by a standard argument (see for example, Stokey, Lucas with Prescott, 1989, Theorem 4.8) $B_{T}$ is also strictly convex. Suppose $B_{t+1}$ is strictly convex. The constraints in problem (11) are linear and, since $B_{t+1}$ is strictly convex so is the objective in problem (11). Thus, $B_{t}$ is also strictly convex. Applying this argument iteratively from period $T-1$ back to period 0 completes the proof.

Proof of Lemma 3: Given the multiplicative nature of the shocks, existence of optimal functions 
$\left\{\varphi_{t}^{*}\left(w_{t}, \cdot\right), \varsigma_{t}^{*}\left(w_{t}, \cdot\right), \omega_{t+1}^{*}\left(w_{t}, \cdot\right)\right\}$ that solve problem $(11)$ when $w \in \mathcal{W}_{t}$ can be established by applying the arguments of Kahn (1993). The existence of functions $\left\{\varphi_{T}^{*}(w, \cdot), v_{T}^{*}(w, \cdot)\right\}$ that solve problem (14) when $w_{t} \in \mathcal{W}_{t+1}$ can be similarly established.

By a standard argument, if $\left(\varphi^{\prime}, \varsigma^{\prime}\right)$ satisfies the period $T$ incentive-compatibility (15), then $\varphi^{\prime}$ and $\varsigma^{\prime}$ are monotone in $\theta$. Hence, $\left(\varphi_{T}^{*}\left(w_{T}, \cdot\right), \varsigma_{T}^{*}\left(w_{T}, \cdot\right)\right)$ are monotone. Similarly, if $\left(\varphi^{\prime}, \varsigma^{\prime}, \omega^{\prime}\right)$ satisfies the incentivecompatibility condition (12), then $\varsigma^{\prime}$ and $d^{\prime}=\varphi^{\prime}+\beta \omega^{\prime}$ are monotone. Hence, for $t<T,\left(d_{t}^{*}\left(w_{t}, \cdot\right), \varsigma_{t}^{*}\left(w_{t}, \cdot\right)\right)$ are monotone, where $d_{t}^{*}\left(w_{t}, \cdot\right)=\varphi_{t}^{*}\left(w_{t}, \cdot\right)+\beta \omega_{t+1}^{*}\left(w_{t}, \cdot\right)$. Let $\left(\varphi^{\prime}(d), \omega^{\prime}(d)\right)$ denote the solution to

$$
\sup _{\left(\varphi^{\prime}, \omega^{\prime}\right): d=\varphi^{\prime}+\beta \omega^{\prime}}\left[C\left(\varphi^{\prime}\right)+q_{t} B_{t+1}\left(\omega^{\prime}\right)\right] .
$$

It is easy to check that $\left(\varphi_{t}^{*}\left(w_{t}, \theta\right), \omega_{t+1}^{*}\left(w_{t}, \theta\right)\right)=\left(\varphi^{\prime}\left(d_{t}^{*}\left(w_{t}, \theta\right)\right), \omega^{\prime}\left(d_{t}^{*}\left(w_{t}, \theta\right)\right)\right)$. The strict convexity of $C$ and $B_{t+1}$ imply that $\left(\varphi^{\prime}(d), \omega^{\prime}(d)\right)$ are strictly increasing. Since $d_{t}^{*}$ is monotone, it then follows that $\varphi_{t}^{*}$ and $\omega_{t+1}^{*}$ are monotone as well.

Construct $y_{t}^{*}, c_{t}^{*}$ and $B_{t+1}^{*}$ by setting $y_{t}^{*}(b, \theta)=Y\left(\varsigma_{t}^{*}\left(B_{t}^{-1}(b), \theta\right)\right), c_{t}^{*}(b, \theta)=C\left(\varphi_{t}^{*}\left(B_{t}^{-1}(b), \theta\right)\right)$ and $B_{t+1}^{*}(b, \theta)=B_{t+1}\left(\omega_{t+1}^{*}\left(B_{t}^{-1}(b), \theta\right)\right)$.

Proof of Lemma 4 Part 1 . The two incentive constraints imply that $\varsigma(\bar{\theta}) \geq \varsigma(\underline{\theta})$ and $\varphi(\underline{\theta}) \geq \varsigma(\bar{\theta})$. Hence, the "upward" incentive constraint: $\varphi(\underline{\theta})+\underline{\theta} \varsigma(\underline{\theta}) \geq \varphi(\bar{\theta})+\underline{\theta} \varsigma(\bar{\theta})$ must hold with equality at the optimum. If not $\varphi(\underline{\theta})>\varphi(\bar{\theta})$, but then the strict convexity of $C$ implies that the planner can reduce her costs by lowering $\varphi(\underline{\theta})$ by $\varepsilon$ and raising $\varphi(\bar{\theta})$ by $\varepsilon \pi(\underline{\theta}) / \pi(\bar{\theta})$. The promise keeping and upward incentive constraint can then be used to eliminate the $\varphi$ variables from the planner's problem. We drop the downward incentive constraint $\varphi(\bar{\theta})+\bar{\theta} \varsigma(\bar{\theta}) \geq \varphi(\underline{\theta})+\bar{\theta} \varsigma(\underline{\theta})$, and consider the following problem:

$$
\sup _{\{\varsigma(\underline{\theta}), \varsigma(\bar{\theta})\}}\left\{C\left(w_{1}-(\bar{\theta}-\underline{\theta}) \varsigma(\bar{\theta}) \pi(\bar{\theta})-\underline{\theta} \varsigma(\underline{\theta})\right)-Y(\varsigma(\underline{\theta}))\right\} \pi(\underline{\theta})+\left\{C\left(w_{1}-E[\theta] \varsigma(\bar{\theta})\right)-Y(\varsigma(\bar{\theta}))\right\} \pi(\bar{\theta}) .
$$

It follows from (66), the convexity of $C$ and $-Y$ and the fact that $C^{\prime}(0)=0$, that there is a critical $A$ such that if $A\left(w_{1}\right) \equiv w_{1}-(\bar{\theta}-\underline{\theta}) \varsigma_{1}^{*}\left(w_{1}, \bar{\theta}\right) \pi(\bar{\theta})>A$, then $\varsigma_{1}^{*}\left(w_{1}, \underline{\theta}\right)=v(\underline{y})$. If $A\left(w_{1}\right)<A, \varsigma_{1}^{*}\left(w_{1}, \underline{\theta}\right)=v(\bar{y})$. Suppose that $A\left(w_{1}\right)>A$, then $C\left(A\left(w_{1}\right)-\underline{\theta} v(\underline{y})\right)-\underline{y}<C\left(A\left(w_{1}\right)-\underline{\theta} v(\bar{y})\right)-\bar{y}$. Also, $w_{1}=A\left(w_{1}\right)+$ $(\bar{\theta}-\underline{\theta}) \varsigma_{1}^{*}\left(w_{1}, \bar{\theta}\right) \pi(\bar{\theta})>A\left(w_{1}\right)$. So, by the convexity of $C, C\left(w_{1}-\underline{\theta} v(\underline{y})\right)-\underline{y}<C\left(w_{1}-\underline{\theta} v(\bar{y})\right)-\bar{y}$. Again by the strict convexity of $C, C\left(w_{1}-E[\theta] v(\underline{y})\right)-\underline{y}<C\left(w_{1}-E[\theta] v(\bar{y})\right)-\bar{y}$. Thus, if $A\left(w_{1}\right)>A$, it follows from (66) that the cost in state $\bar{\theta}$ is lower if $\varsigma(\bar{\theta})=v(\underline{y})$. The cost in state $\underline{\theta}$ is also lower if $\varsigma(\bar{\theta})=v(\underline{y})$. So in fact $\varsigma_{1}^{*}\left(w_{1}, \bar{\theta}\right)=v(\underline{y})$. It follows that there exists a critical $\bar{w}_{1}=A+(\bar{\theta}-\underline{\theta}) v(\underline{y}) \pi(\bar{\theta})$, such that for 
$w_{1}>\bar{w}_{1}, \varsigma_{1}^{*}\left(w_{1}, \underline{\theta}\right)$ and $\varsigma_{1}^{*}\left(w_{1}, \bar{\theta}\right)$ equal $v(\underline{y})$, for $w_{1}<\bar{w}_{1}, \varsigma_{1}^{*}\left(w_{1}, \underline{\theta}\right)$ equals $v(\bar{y})$. For $w_{1}<\bar{w}_{1}, \varsigma_{1}^{*}\left(w_{1}, \bar{\theta}\right)$ solves

$$
\sup _{\{\varsigma(\bar{\theta})\}}\left\{C\left(w_{1}-(\bar{\theta}-\underline{\theta}) \varsigma(\bar{\theta}) \pi(\bar{\theta})-\underline{\theta} v(\bar{y})\right)\right\} \pi(\underline{\theta})+\left\{C\left(w_{1}-E[\theta] \varsigma(\bar{\theta})\right)-Y(\varsigma(\bar{\theta}))\right\} \pi(\bar{\theta}) .
$$

It follows easily from the convexity of $C$ and $-Y$ that there exists a critical $\underline{w}_{1} \in \mathbb{R} \cup\{-\infty\}$, such that for $w_{1}>\underline{w}_{1}, \varsigma^{*}\left(w_{1}, \bar{\theta}\right)=v(\underline{y})$, and for $w_{1}<\underline{w}_{1}, \varsigma_{1}^{*}\left(w_{1}, \bar{\theta}\right)=v(\bar{y})$. (The set $w_{1}<\underline{w}_{1}$ may be empty). It follows from the above discussion that the solution to (66) satisfies the upwards incentive constraint with equality and $\varsigma_{1}^{*}\left(w_{1}, \cdot\right)$ is non-decreasing. Hence, this solution satisfies the downwards incentive constraint and solves the original component planner's problem.

Part 2. It follows from the previous part that

$$
B_{1}(w)=\left\{\begin{aligned}
B_{11}\left(w_{1}\right)=C\left(w_{1}-E[\theta] v(\bar{y})\right)-\bar{y} & \text { for } & w_{1}<\underline{w}_{1} \\
B_{12}\left(w_{1}\right)=\left\{C\left(w_{1}-(\bar{\theta}-\underline{\theta}) v(\underline{y}) \pi(\bar{\theta})-\underline{\theta} v(\bar{y})\right)-\bar{y}\right\} \pi(\underline{\theta}) & \text { for } & w_{1} \in\left(\underline{w}_{1}, \bar{w}_{1}\right) \\
\quad+\left\{C\left(w_{1}-E[\theta] v(\underline{y})\right)-\underline{y}\right\} \pi(\bar{\theta}) & & \\
B_{13}\left(w_{1}\right)=C\left(w_{1}-E[\theta] v(\underline{y})\right)-\underline{y} & \text { for } & w_{1}>\bar{w}_{1} .
\end{aligned}\right.
$$

Each $B_{1 i}$ is strictly convex and differentiable. Hence, $B_{1}$ is piecewise strictly convex and differentiable except at $\underline{w}_{1}$ and $\bar{w}_{1}$.

Part 3. Let $B_{0 i}\left(w_{0}\right)=\sup C\left(w_{0}-\beta w_{1}\right)+q B_{1 i}\left(w_{1}\right)$. Let $w_{1 i}^{*}\left(w_{0}\right)$ denote the solutions to these problems, where $C^{\prime}\left(w_{0}-\beta w_{1 i}^{*}\left(w_{0}\right)\right)=q / \beta B_{1 i}^{\prime}\left(w_{1 i}^{*}\left(w_{0}\right)\right)$. Clearly, $B_{11}^{\prime}>B_{12}^{\prime}>B_{13}^{\prime}$, and so $w_{11}^{*}\left(w_{0}\right)<w_{12}^{*}\left(w_{0}\right)<$ $w_{13}^{*}\left(w_{0}\right)$. Thus, $B_{01}^{\prime}\left(w_{0}\right)=C^{\prime}\left(w_{0}-\beta w_{11}^{*}\left(w_{0}\right)\right)>C^{\prime}\left(w_{0}-\beta w_{12}^{*}\left(w_{0}\right)\right)=B_{02}^{\prime}\left(w_{0}\right)$. Also, $w_{11}^{*}\left(w_{0}\right)$ is strictly increasing, and eventually, $w_{11}^{*}\left(w_{0}\right)>\underline{w}_{1}$. At this point, $B_{01}\left(w_{0}\right)=C\left(w_{0}-\beta w_{1}^{*}\left(w_{0}\right)\right)+q B_{11}\left(w_{1}^{*}\left(w_{0}\right)\right)=$ $C\left(w_{0}-\beta w_{1}^{*}\left(w_{0}\right)\right)+q B_{12}\left(w_{1}^{*}\left(w_{0}\right)\right) \geq C\left(w_{0}-\beta w_{2}^{*}\left(w_{0}\right)\right)+q B_{12}\left(w_{2}^{*}\left(w_{0}\right)\right)$. Thus, there exists a critical $\underline{w}_{0}^{12}$ such that for $w_{0}>$ (resp. $\left.<\right) \underline{w}_{0}^{12}, B_{02}\left(w_{0}\right)<B_{01}\left(w_{0}\right)$. By identical reasoning, there exists a critical $\bar{w}_{0}^{23}$ such that for $w_{0}>($ resp. $<) \bar{w}_{0}^{23}, B_{03}\left(w_{0}\right)<B_{02}\left(w_{0}\right)$ and a critical $\bar{w}_{0}^{13}$ such that for $w_{0}>($ resp. $<) \bar{w}_{0}^{13}$, $B_{03}\left(w_{0}\right)<B_{01}\left(w_{0}\right)$. Let $\underline{w}_{0}=\min \left(\underline{w}_{0}^{12}, \underline{w}_{0}^{13}\right)$ and $\bar{w}_{0}=\min \left(\underline{w}_{0}^{23}, \underline{w}_{0}^{13}\right)$. Then, for $w<\underline{w}_{0}, \omega^{*}=w_{11}^{*}$, for $w \in\left(\underline{w}_{0}, \bar{w}_{0}\right), \omega^{*}=w_{12}^{*}$, and for $w>\bar{w}_{0}, \omega^{*}=w_{13}^{*}$. Thus, $\omega^{*}$ is increasing.

Proof of Lemma 6: By (53), if the agent saves $b_{1}^{*}$, she attains the consumption level $c_{1}^{*}(\bar{y})$ if she chooses $\bar{y}$ and $c_{1}^{*}(\underline{y})$ if she chooses $\underline{y}$. The definitions of $\underline{T}_{1}$ and $\bar{T}_{1}$ imply $q u^{\prime}\left(c_{0}^{*}\right)=\beta\left(1-\bar{T}_{1}\right) u^{\prime}\left(c_{1}^{*}(\underline{\theta})\right)$ and $q u^{\prime}\left(c_{0}^{*}\right)=\beta\left(1-\underline{T}_{1}\right) u^{\prime}\left(c_{1}^{*}(\bar{\theta})\right)$. Thus, independently of the agent's labour choice in period 1 , it is optimal for the agent to save $b_{1}^{*}$. It then follows from the component planner's incentive-compatibility condition 
that the agent will choose $\left(c_{1}^{*}(\underline{\theta}), \bar{y}\right)$ when her shock equals $\underline{\theta}$ and $\left(c_{1}^{*}(\bar{\theta}), \underline{y}\right)$ otherwise. Hence, $a^{*}$ can be implemented in a market economy with the tax function (54).

Now, suppose that the tax function $T(b, y)=T_{0}(y)+T_{1}(y) b$ implements $a^{*}$ in the market economy and that $T_{1}(\underline{y})<\underline{T}_{1}$. Then, as in the proof of the previous lemma, the agent can save $b_{1}^{*}$ and select $\underline{y}$ regardless of her shock. This is feasible and delivers the same payoff, $w_{0}$, to the agent as $a^{*}$. However, since $T_{1}(\underline{y})<\underline{T}_{1}, q u^{\prime}\left(c_{0}^{*}\right)<\beta\left(1-T_{1}(\underline{y})\right) u^{\prime}\left(c_{1}^{*}(\bar{\theta})\right)$, so that the agent can do even better and obtain a payoff above $w_{0}$ by saving slightly more than $b_{1}^{*}$, and selecting an effort of $\underline{y}$ regardless of her shock. It follows that if $T(b, y)$ implements $a^{*}$ then $T_{1}(\underline{y}) \geq \underline{T}_{1}$. Similarly, if $T_{1}(\underline{y})>\underline{T}_{1}$, the agent can improve on the planner's solution by saving slightly less than $b_{1}^{*}$ and choosing $\underline{y}$ regardless of her shock. Thus, $T_{1}(\underline{y})=\underline{T}_{1}$. It then follows from (48), that the agent's $a^{*}$ is consistent with the agent's Euler equation only if $T_{1}(\bar{y})=\bar{T}_{1}$.

Proof of Lemma 9: Define $K(\widehat{\theta})=\bar{v} \int_{\underline{\theta}}^{\widehat{\theta}}(\widehat{\theta}-\theta) \pi(\theta) d \theta-\underline{v} \int_{\widehat{\theta}}^{\bar{\theta}}(\theta-\widehat{\theta}) \pi(\theta) d \theta$. The promise keeping and incentive-compatibility constraints imply that:

$$
\underline{\varphi}_{1}=\frac{1}{\beta}\left(w_{0}-\varphi_{0}\right)+K(\widehat{\theta})-\widehat{\theta} \underline{v} \quad \text { and } \quad \bar{\varphi}_{1}=\frac{1}{\beta}\left(w_{0}-\varphi_{0}\right)+K(\widehat{\theta})-\widehat{\theta} \bar{v} .
$$

Let $\widehat{\theta}^{\circ}\left(w_{0}\right)=\widehat{\theta}_{1}^{*}\left(\omega^{*}\left(w_{0}\right)\right), \underline{\varphi}_{1}^{\circ}\left(w_{0}\right)=\bar{\varphi}_{1}^{\circ}\left(\omega^{*}\left(w_{0}\right)\right)$ and $\bar{\varphi}_{1}^{\circ}\left(w_{0}\right)=\bar{\varphi}_{1}^{*}\left(\omega^{*}\left(w_{0}\right)\right)$. Suppose that $w_{0}^{2}>w_{0}^{1}$ and that $\tilde{\theta}=\widehat{\theta}^{\circ}\left(w_{0}^{2}\right)=\widehat{\theta}^{\circ}\left(w_{0}^{2}\right)$. The first order conditions for the component planner's problem yield at each $w_{0}^{i}, i=1,2$,

$$
C^{\prime}\left(\varphi_{0}^{*}\right)=\frac{q}{\beta}\left[C^{\prime}\left(\bar{\varphi}_{1}^{\circ}\right) \Pi(\widetilde{\theta})+C^{\prime}\left(\underline{\varphi}_{1}^{\circ}\right)(1-\Pi(\widetilde{\theta}))\right] .
$$

(67), (68) and the strict convexity of $C$ imply that $\varphi_{0}^{*}\left(w_{0}^{2}\right)>\varphi_{0}^{*}\left(w_{0}^{2}\right), \underline{\varphi}_{1}^{\circ}\left(w_{0}^{2}\right)>\underline{\varphi}_{1}^{\circ}\left(w_{0}^{1}\right)$ and $\bar{\varphi}_{1}^{\circ}\left(w_{0}^{2}\right)>$ $\bar{\varphi}_{1}^{\circ}\left(w_{0}^{1}\right)$. The first order conditions from the component planner's problem also imply that at each $w_{0}^{i}$, $i=1,2$,

$$
\left[\left\{C\left(\bar{\varphi}_{1}^{\circ}\right)-Y(\bar{v})\right\}-\left\{C\left(\underline{\varphi}_{1}^{\circ}\right)-Y(\underline{v})\right\}\right] \rho(\widetilde{\theta})+\left[C^{\prime}\left(\bar{\varphi}_{1}^{\circ}\right) \Delta v(1-\Pi(\widetilde{\theta}))-C^{\prime}\left(\underline{\varphi}_{1}^{\circ}\right) \Delta v \Pi(\widetilde{\theta})\right]=0 .
$$

The first term above is negative: the component planner obtains a higher quantity of net resources when the agent exerts higher effort. Consequently, the second term is positive. Now incentive-compatibility implies: $\bar{\varphi}^{\circ}\left(w_{0}^{i}\right)-\underline{\varphi}^{\circ}\left(w_{0}^{i}\right)=\tilde{\theta} \Delta v$, where $\Delta v=\underline{v}-\bar{v}>0$. The convexity of $C$ then implies that $C\left(\bar{\varphi}_{1}^{\circ}\left(w_{0}^{2}\right)\right)$ $-C\left(\underline{\varphi}_{1}^{\circ}\left(w_{0}^{2}\right)\right)>C\left(\bar{\varphi}_{1}^{\circ}\left(w_{0}^{1}\right)\right)-C\left(\underline{\varphi}_{1}^{\circ}\left(w_{0}^{1}\right)\right)$, so that the first term in the above expression increases across the two utility promise levels $w_{0}^{1}$ and $w_{0}^{2}$. Next let $H(\varphi)=\left[C^{\prime}(\varphi+\widetilde{\theta} \Delta v) \Delta v(1-\Pi(\widetilde{\theta}))-C^{\prime}(\varphi) \Delta v \Pi(\widetilde{\theta})\right]$. 
Then

$$
\begin{aligned}
H^{\prime}(\varphi) & =\left[C^{\prime \prime}(\varphi+\widetilde{\theta} \Delta v) \Delta v(1-\Pi(\widetilde{\theta}))-C^{\prime \prime}(\varphi) \Delta v \Pi(\widetilde{\theta})\right] \\
& =\frac{C^{\prime \prime}(\varphi+\widetilde{\theta} \Delta v)}{C^{\prime}(\varphi+\widetilde{\theta} \Delta v)}\left[C^{\prime}(\varphi+\widetilde{\theta} \Delta v) \Delta v(1-\Pi(\widetilde{\theta}))-\frac{C^{\prime \prime}(\varphi)}{C^{\prime}(\varphi)} \frac{C^{\prime}(\varphi+\tilde{\theta} \Delta v)}{C^{\prime \prime}(\varphi+\widetilde{\theta} \Delta v)} C^{\prime}(\varphi) \Delta v \Pi(\widetilde{\theta})\right] \\
& >\frac{C^{\prime \prime}(\varphi+\widetilde{\theta} \Delta v)}{C^{\prime}(\varphi+\widetilde{\theta} \Delta v)}\left[C^{\prime}(\varphi+\widetilde{\theta} \Delta v) \Delta v(1-\Pi(\widetilde{\theta}))-C^{\prime}(\varphi) \Delta v \Pi(\widetilde{\theta})\right]>0 .
\end{aligned}
$$

Here, the first inequality follows from the assumption in the lemma and the second from the fact that

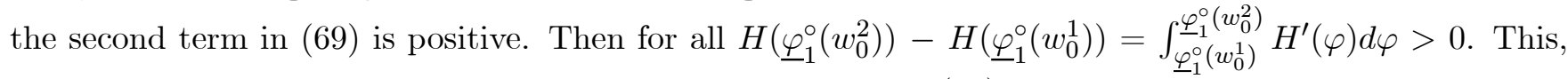
however, implies that the second term on the left hand side of (69) is increasing. But then both terms on the left hand side of (69) are higher at $w_{0}^{2}$ than $w_{0}^{1}$. This contradicts (69) holding at both $w_{0}^{2}$ than $w_{0}^{1}$.

If $b_{1}$ and $b_{1}^{\prime}$ are in $\mathcal{B}_{1}^{*}$, then there exists a $w_{0}$ and a $w_{0}^{\prime}$ such that $b_{1}=B_{1}\left(\omega^{*}\left(w_{0}\right)\right)$ and $b_{1}^{\prime}=B_{1}\left(\omega^{*}\left(w_{0}^{\prime}\right)\right)$. Since $b_{1} \neq b_{1}, w_{0} \neq w_{0}^{\prime}$ and $\theta_{1}^{*}\left(b_{1}\right)=\theta^{\circ}\left(w_{0}\right) \neq \theta^{\circ}\left(w_{0}^{\prime}\right)=\theta_{1}^{*}\left(b_{1}^{\prime}\right)$.

Proof of Proposition 3: Truncate the infinite-period market economy at $T<\infty$. Set the agent's period $T+1$ value function in the truncated economy to $\widehat{V}_{T+1}=B_{T+1}^{-1}$. Subdivide each period $t \in$ $\{0, \ldots, T\}$ into two sub-periods. In the first the agent exerts effort $y_{t}$ and obtains some after-tax resources $x_{t}=b_{t}+y_{t}-T_{t}\left(b_{t}, y_{t}\right)$. In the second, the agent allocates $x_{t}$ between consumption $c_{t}$ and savings $b_{t+1}$. Each period of the component planner's problem can be similarly sub-divided. In the first sub-period, the agent makes a report and the component planner allocates utility from effort and an interim utility promise; in the second, the planner allocates the interim utility promise between utility from consumption and a continuation utility promise. By repeated application of the argument in the proof of Proposition 1 , beginning in period $T$, a sequence of tax functions $\left\{T_{t}\right\}_{t=0}^{T}$ and borrowing limits $\left\{\underline{b}_{t+1}\right\}_{t=0}^{T-1}$ can be constructed such that confronted with these sequences, it is optimal for an agent with initial wealth $B_{0}\left(w_{0}\right)$ to choose the same period $T$-truncated allocation, $\left\{c_{t}, y_{t}\right\}_{t=0}^{T}$ as is awarded to an agent with a $w_{0}$-utility promise by the component planner.

By successively increasing $T$, a sequence of tax functions $\left\{T_{t}\right\}_{t=0}^{\infty}$ and borrowing limits $\left\{\underline{b}_{t+1}\right\}_{t=0}^{\infty}$ can be constructed which, along with $\left\{G_{t}\right\}_{t=0}^{\infty}$ and $\Lambda_{0}$ define a market economy. Set $f=B_{0}$. If an agent with an initial quantity of claims $B_{0}\left(w_{0}\right)$ selects $z^{*}\left(w_{0}\right)$, the allocation obtained by a $w_{0}$-promise agent in the component economy, then she too receives a payoff of $w_{0}$. Suppose there is some alternative allocation available to the agent in the constructed market economy that gives a payoff of $\widetilde{U}^{\infty}>w_{0}+\varepsilon>w_{0}$. Let $\widetilde{U}^{T}$ denote the payoff earned from this allocation over the initial $T$ periods, and let $\widetilde{b}_{T+1}$ denote the 
agent's savings at date $T$ under this allocation. Similarly, let $U^{T}$ be the agent's payoff from $z^{*}\left(w_{0}\right)$ over the initial $T$ periods and let $b_{T+1}$ be the agent's savings at date $T$ under this allocation. Now since agents choose to select the component planner allocation in the truncated economy: $U^{T}\left(w_{0}\right)+\beta^{T} E \widehat{V}_{T+1}\left(b_{T+1}\right) \geq$ $\widetilde{U}^{T}+\beta^{T} E \widehat{V}_{T+1}\left(\widetilde{b}_{T+1}\right)$ Hence, since the agent's utility functions are bounded, $w_{0}=\lim _{T \rightarrow \infty}\left[U^{T}\left(w_{0}\right)+\right.$ $\left.\beta^{T} E \widehat{V}_{T+1}\left(b_{T+1}\right)\right] \geq \lim _{T \rightarrow \infty}\left[\widetilde{U}^{T}+\beta^{T} E \widehat{V}_{T+1}\left(\widetilde{b}_{T+1}\right)\right]=\widetilde{U}^{\infty}>w_{0}+\varepsilon$. This is a contradiction. It then follows that in the untruncated economy, the agent with initial wealth $B_{0}\left(w_{0}\right)$ selects the allocation obtained by an agent with an initial utility promise of $w_{0}$. Condition 2 in the implementation definition is satisfied. 


\section{References}

[1] Aiyagari, S. R. 1994. Uninsured idiosyncratic risk and aggregate saving. Quarterly Journal of Economics 109:659-84.

[2] Albanesi, S. and C. Sleet. 2004. Notes on dynamic optimal taxation with private information. Mimeo. University of Iowa.

[3] Atkeson, A. and R. Lucas. 1992. On efficient distribution with private information. The Review of Economic Studies 59:427-453.

[4] Atkeson, A. and R. Lucas. 1995. Efficiency and equality in a simple model of efficient unemployment insurance. Journal of Economic Theory 66:64-88.

[5] Bewley, T. 1986. Stationary monetary equilibrium with a continuum of independently fluctuating consumers. In W. Hildenbrand and A. Mas-Colell (eds.) Contributions to Mathematical Economics in Honor of Gerard Debreu. Amsterdam: North Holland.

[6] Brito, D., J. Hamilton, S. Slutsky and J. Stiglitz. 1991. Dynamic optimal taxation with government commitment. Journal of Public Economics 44:15-35.

[7] Chari, V.V., and P. Kehoe. 1999. Optimal Fiscal and Monetary Policy. Handbook of Macroeconomics, Volume 1, Elsevier.

[8] da Costa, C. and I. Werning. 2001. On the optimality of the Friedman rule with heterogeneous agents and non-linear income taxation. Unpublished paper. University of Chicago.

[9] Diamond, P. 1998. Optimal taxation: An example with a U-shaped pattern of optimal marginal tax rates. American Economic Review 88:83-95.

[10] Diamond, P. and J. Mirrlees. 1978. A model of social insurance with variable retirement. Journal of Public Economics 10:295-336.

[11] Doepke, M. and R. Townsend. 2002. Dynamic Mechanism Design with Hidden Income and Hidden Actions. Manuscript. UCLA and The University of Chicago. 
[12] Fernandes, A. and C. Phelan. 2000. A recursive formulation for repeated agency with history dependence. Journal of Economic Theory 91:223-247.

[13] Golosov, M., N. Kocherlakota and A. Tsyvinski. 2003. Optimal indirect and capital taxation. Review of Economic Studies 70:569-587.

[14] Golosov, M. and A. Tsyvinski. 2003. Designing optimal disability insurance. Federal Reserve Bank of Minneapolis. Working Paper 628.

[15] Green, E. 1987. Lending and the smoothing of uninsurable income. In Contractual arrangements for international trade eds. E. Prescott and N. Wallace. Minnesota University Press, Minnesota.

[16] Heathcote, J, K. Storesletten, and G. Violante. 2003. The Macroeconomic Implications of Rising Wage Inequality in the US. Manuscript. New York University.

[17] Huggett, M. 1993. The risk free rate in heterogeneous-agent, incomplete-insurance economies. Journal of Economic Dynamics and Control 17:953-969.

[18] Judd, K. 1985. The law of large numbers with a continuum of IID random variables. Journal of Economic Theory 35:19-25.

[19] Kahn, C. 1993. Existence and characterisation of optimal employment contracts on a continuous state space. Journal of Economic Theory 59:122-144.

[20] Kocherlakota, N. 2003. Zero expected wealth taxes: A Mirrlees approach to dynamic optimal taxation. Federal Reserve Bank of Minneapolis WP.

[21] Ligon, E. 1998. Risk-sharing and information in village economies. The Review of Economic Studies 65: $847-864$

[22] Mirrlees, J. 1971. An exploration in the theory of optimum income taxation. The Review of Economic Studies 38:175-208.

[23] Phelan, C. 1994. Incentives and aggregate shocks. The Review of Economic Studies 61:681-700.

[24] Phelan, C. and R. Townsend. 1991. Computing multi-period, information constrained optima. The Review of Economic Studies 58:853-881. 
[25] Rogerson, W. 1985. Repeated moral hazard. Econometrica 53:69-76.

[26] Saez, E. 2001. Using elasticities to derive optimal income tax rates. The Review of Economic Studies 68:205-229.

[27] Salanié, B. 1997. The economics of contracts: a primer. MIT Press: Cambridge, Mass.

[28] Seade, J. 1977. On the Shape of Optimal Tax Schedules. Journal of Public Economics, 7(2): 203-235.

[29] Stokey, N., R. Lucas with E. Prescott. 1989. Recursive methods in economic dynamics. Harvard University Press: Cambridge, Mass.

[30] Townsend, R. 1995. Consumption Insurance: An Evaluation of Risk-Bearing Systems in Low-Income Economies. Journal of Economic Perspectives, 9(3), 83-102. 


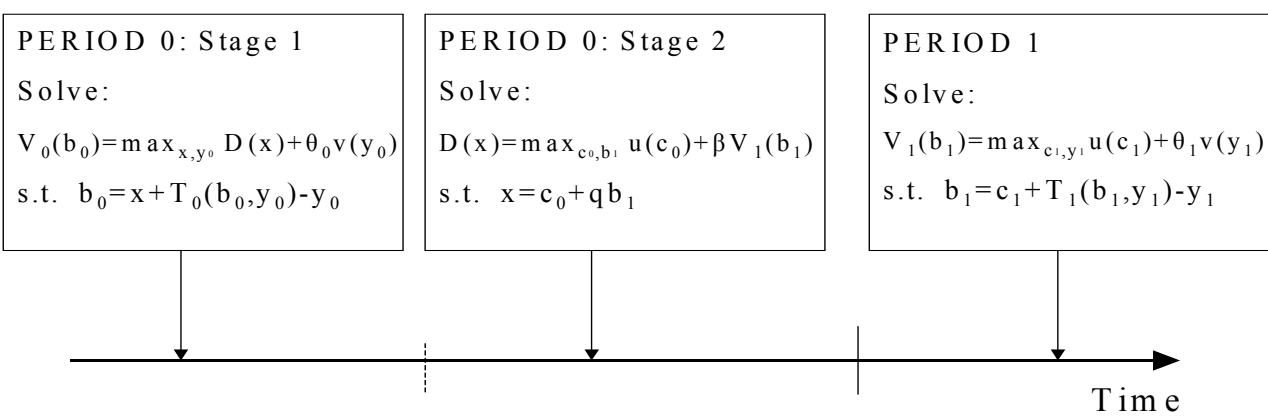

Period 0

Period 1

Figure A1: Timeline for the market economy.

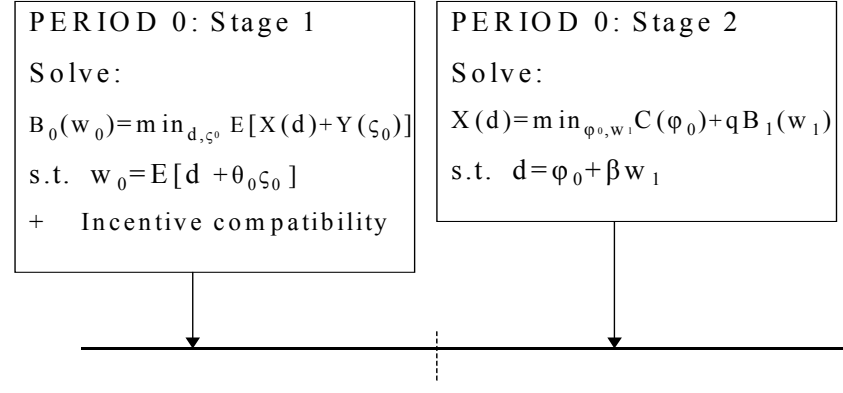

Period 0
PER IO D 1

Solve:

$\mathrm{B}_{1}\left(\mathrm{w}_{1}\right)=\mathrm{min}_{\varphi 1, \zeta_{1}} \mathrm{E}\left[\mathrm{C}\left(\varphi_{1}\right)+\mathrm{Y}\left(\varsigma_{1}\right)\right]$

s.t. $\mathrm{w}_{1}=\mathrm{E}\left[\varphi_{1}+\theta_{1} \varsigma_{1}\right]$

$+\quad$ Incentive compatibility

Figure A2: Timeline for the component planner economy. 